TRANSACTIONS OF THE

AMERICAN MATHEMATICAL SOCIETY

Volume 352, Number 4, Pages 1913-1934

S 0002-9947(99)02309-0

Article electronically published on December 10, 1999

\title{
PERIODIC GROUPS COVERED BY TRANSITIVE SUBGROUPS OF FINITARY PERMUTATIONS OR BY IRREDUCIBLE SUBGROUPS OF FINITARY TRANSFORMATIONS
}

\author{
FELIX LEINEN AND ORAZIO PUGLISI
}

\begin{abstract}
Let $\mathfrak{X}$ be either the class of all transitive groups of finitary permutations, or the class of all periodic irreducible finitary linear groups. We show that almost primitive $\mathfrak{X}$-groups are countably recognizable, while totally imprimitive $\mathfrak{X}$-groups are in general not countably recognizable. In addition we derive a structure theorem for groups all of whose countable subsets are contained in totally imprimitive $\mathfrak{X}$-subgroups. It turns out that totally imprimitive $p$-groups in the class $\mathfrak{X}$ are countably recognizable.
\end{abstract}

\section{INTRODUCTION}

A class $\mathfrak{G}$ of groups is said to be countably recognizable if every group, all of whose countable subsets are contained in countable $\mathfrak{G}$-subgroups, is itself a $\mathfrak{G}$ group. Many examples of such classes are discussed in [21, Section 8.3]. In the present work we are concerned with countable recognizability for transitive finitary permutation groups and for periodic irreducible finitary linear groups. Recall that a group is said to be a finitary permutation group if it is isomorphic to a subgroup of $\operatorname{FSym}(\Omega)$, the group of those permutations of some set $\Omega$ which fix all but finitely many elements. Correspondingly, a group is said to be finitary $\mathbb{F}$-linear if it is isomorphic to a subgroup of $\mathrm{FGL}_{\mathbb{F}}(V)$, the group of those invertible $\mathbb{F}$-linear transformations of some $\mathbb{F}$-vector space $V$ which act like the identity on a subspace of finite codimension in $V$. Note that $\operatorname{FSym}(\Omega)$ is periodic and finitary linear on the natural $\mathbb{F}$-module $\mathbb{F} \Omega$. A survey about features of finitary linear groups is given in 20 .

By a classical theorem of A. I. Mal'cev [14, Theorem IV], a group is linear of degree $n$ if and only if all its finitely generated subgroups are linear of degree $n$. Later, it was shown by J.I. Hall [4, Theorem 1] that simple finitary linear groups are countably recognizable. This result was extended by the authors to periodic primitive finitary linear groups [13, Theorem A]. Here we made use of Hall's classification of the non-linear periodic simple finitary linear groups [6], [5], and of the fact that the commutator subgroup of a non-linear periodic primitive finitary linear group is simple [13, Theorem B]. In their recent paper [7], K. K. Hickin

Received by the editors February 10, 1997 and, in revised form, October 22, 1997.

1991 Mathematics Subject Classification. Primary 20B07, 20E25, 20F50, 20H20; Secondary 03C20, 20E22.

Key words and phrases. Countable recognizability, finitary linear groups, finitary permutation groups, locally finite groups, wreath products, ultraproducts.

Each of the two authors would like to thank the university of his coauthor for inviting him to a visit, during which essential parts of the work on this paper could be carried out. 
and R. E. Phillips derived partial results about countable recognizability in certain classes of totally imprimitive finitary permutation groups. In the present article we shall complete all these investigations with the following general treatment.

Notation. Throughout, let $\mathfrak{X}$ be either the class of all transitive groups of finitary permutations, or the class of all periodic irreducible finitary linear groups.

An $\omega$-cover of the group $G$ is a family of countable subgroups of $G$ such that each countable subset of $G$ is contained in a subgroup from this family. The notions totally imprimitive and almost primitive, which apply to transitive groups of finitary permutations as well as to irreducible groups of finitary transformations, are explained in Section 2. Note that totally imprimitive $\mathfrak{X}$-groups are always countable (see Section 2).

Theorem A. Almost primitive $\mathfrak{X}$-groups are countably recognizable.

Theorem B. There exist uncountable groups which admit neither a faithful transitive finitary permutation representation nor a faithful irreducible finitary linear representation, although they contain an $\omega$-cover of totally imprimitive $\mathfrak{X}$-subgroups.

In fact, we shall construct a huge variety of such groups, even with prescribed composition factors. On the other hand, we shall complement Theorem B with a very precise structure theorem for groups which contain an $\omega$-cover of totally imprimitive $\mathfrak{X}$-subgroups.

Theorem C. Let $G$ be a group with an $\omega$-cover of totally imprimitive $\mathfrak{X}$-subgroups. Then $G$ embeds as a transitive resp. irreducible subgroup into the unrestricted wreath product $W=S \mathrm{Wr}_{\omega} \operatorname{FSym}(\omega)$, where $S$ is a finite group resp. an irreducible subgroup of $\mathrm{GL}_{\mathbb{K}}(U)$ for some finite-dimensional vector space $U$ over an algebraically closed field $\mathbb{K}$. Here, $W$ acts naturally on the set $S \times \omega$ resp. on the vector space $U \otimes \omega$. Further, the following three properties hold.

(a) The image $G \pi$ of the projection $\pi$ of $G$ into the top group of $W$ is a totally imprimitive subgroup of $\operatorname{FSym}(\omega)$.

(b) $G$ is contained in $W_{0} \cdot C$, where $W_{0}$ denotes the restricted wreath product $S \operatorname{wr}_{\omega} \operatorname{FSym}(\omega)$, and where $C$ is the group of all base group functions $f: \omega \rightarrow \zeta_{1}(S)$ which, for every proper $G \pi$-block $\Omega_{0}$ in $\omega$, are constant on all but finitely many $G \pi$-translates of $\Omega_{0}$, resp. act like a scalar on all but finitely many $G$-translates of $U \otimes \Omega_{0}$.

(c) In particular, $G^{\prime}$ is countable, being a subgroup of $W_{0}$, and $C \cap G$ is an abelian normal subgroup of countable index in $G$.

The properties recorded in Theorem $\mathrm{C}$ are reflected in the examples constructed to show Theorem B. In Section 7 we shall also derive sufficient conditions which ensure countable recognizability in the totally imprimitive case. As an amazing consequence we obtain

Theorem D. Every p-group with an $\omega$-cover of totally imprimitive $\mathfrak{X}$-subgroups is a totally imprimitive $\mathfrak{X}$-group.

In particular, no $p$-group in the class $\mathfrak{X}$ is $L_{\omega_{1}, \omega}$-equivalent to an uncountable group.

[11, Example 3.4] shows, that finitary permutation $p$-groups and unipotent finitary linear $p$-groups are not countably recognizable. In fact, the direct product of the above example and any of the groups constructed in the proof of Theorem B 
has no faithful finitary linear representation at all, although each of its countable subgroups is a group of finitary permutations! (Cf. Section 5.4.) For any pair of primes $p \neq q$ we even obtain periodic locally solvable $\{p, q\}$-groups with this property. Therefore just the following question remains open.

Question. Are finitary linear $p$-groups over fields of characteristic $\neq p$ countably recognizable?

Note that such groups are subdirect products of irreducible finitary linear $p$ groups over a fixed field of coprime characteristic. In his recent work [24], B. A. F. Wehrfritz shows that the answer to the Question is affirmative for hypercentral groups of central height $\omega$.

\section{BACKGROUND INFORMATION}

This section is a résumé of well-known properties and techniques which we intend to use in our proofs without further reference. Note first that every periodic group of finitary permutations or finitary linear transformations is locally finite.

2.1. Finitary Permutation Groups (see [17, [18]). Let $G$ be a transitive subgroup of $\operatorname{FSym}(\Omega)$, where $\Omega$ is infinite. The support $\operatorname{supp}_{\Omega} A$ of a subset $A$ of $G$ is the set of all those elements from $\Omega$ which are actually moved by $A$. Since $G$ contains just elements of finite support, any proper $G$-block in $\Omega$ is finite. Hence one of the following three cases occurs.

(1) $\Omega$ contains no proper $G$-block, that is, $G$ is primitive. By a well-known theorem of H. Wielandt [26, Satz 9.4], the group $G$ is then either $\operatorname{FSym}(\Omega)$ or the alternating group $\operatorname{Alt}(\Omega)$.

(2) There exists a maximal proper $G$-block $\Omega_{0}$ in $\Omega$. Then the induced action of $G$ on the corresponding system $\Delta$ of imprimitivity in $\Omega$ is primitive, whence $G$ is said to be almost primitive. If we identify $\Omega$ in the right way with $\Omega_{0} \times \Delta$, then $G$ becomes a subgroup of the restricted wreath product $W=S_{0} \operatorname{wr}_{\Delta} \operatorname{FSym}(\Delta)$, where $S_{0}$ denotes the subgroup of $\operatorname{Sym}\left(\Omega_{0}\right)$ induced from the setwise stabilizer of $\Omega_{0}$ in $G$. We shall frequently replace the action of $S_{0}$ on $\Omega_{0}$ by the right regular action of $S_{0}$ on itself. In this way, $G$ and $W$ become almost primitive subgroups of $\operatorname{FSym}\left(S_{0} \times \Delta\right)$.

(3) There are proper $G$-blocks in $\Omega$, but no maximal ones. Then $\Omega$ is countable as the union of an ascending chain $\left\{\Omega_{n}\right\}_{n \in \omega}$ of proper $G$-blocks. In particular, $G$ is countable too. In this case $G$ is said to be totally imprimitive. Let $\Delta_{0}=\Omega_{0}$, and for every $n \in \omega$, let $\Delta_{n+1}$ be the system of imprimitivity in $\Omega_{n+1}$ containing the block $\Omega_{n}$. If we identify $\Omega$ in the right way with the restricted product

$$
\left\{\left(\Lambda_{n}\right)_{n \in \omega} \in \prod_{n \in \omega} \Delta_{n} \mid \Lambda_{n}=\Omega_{n} \text { for all but finitely many } n \in \omega\right\},
$$

and if we let $S_{n}$ denote the subgroup of $\operatorname{Sym}\left(\Delta_{n}\right)$ induced from the setwise stabilizer of $\Omega_{n}$ in $G$, then $G$ becomes a subgroup of the restricted wreath product $\mathrm{wr}_{n \in \omega} S_{n}$. This wreath product is the direct limit of the iterated wreath products $G_{n}=S_{0}$ wr $\cdots$ wr $S_{n}$ with respect to the canonical embeddings of $G_{n}$ onto the component of the base group of $G_{n+1}$ corresponding to $\Omega_{n}$.

In all three cases, the derived subgroup $G^{\prime}$ of $G$ has non-trivial intersection with every proper normal subgroup of $G$, and $G^{\prime}$ is the unique minimal transitive normal subgroup of $G$. In particular, $G^{\prime}$ is perfect. In modification of the usual terminology we call $G$ almost primitive if it is not totally imprimitive. 
2.2. Finitary Linear Groups (see [19], [15]). Let $G$ be a periodic irreducible subgroup of $\operatorname{FGL}_{\mathbb{F}}(V)$, where $\operatorname{dim}_{\mathbb{F}} V$ is infinite. Suppose that $G$ is imprimitive. Any proper $G$-block $V_{0}$ in $V$ is finite-dimensional, and $G$ permutes the corresponding system $\Delta$ of imprimitivity transitively and finitarily. If we identify $V$ in the right way with the $\mathbb{F}$-vector space $V_{0} \otimes \Delta=\bigoplus_{\delta \in \Delta} V_{0} \otimes \delta$, then $G$ becomes a subgroup of the restricted wreath product $S_{0} \operatorname{wr}_{\Delta} \operatorname{FSym}(\Delta)$, where $S_{0}$ denotes the subgroup of $\mathrm{GL}_{\mathbb{F}}\left(V_{0}\right)$ induced from the normalizer of $V_{0}$ in $G$. Note that $S_{0}$ acts irreducibly on $V_{0}$.

Depending on the way in which $G$ acts on $\Delta$, the group is said to be almost primitive, respectively totally imprimitive. The unipotent radical of $G$ is trivial, and every normal subgroup of $G$ is completely reducible. Moreover, $G^{\prime}$ has nontrivial intersection with every proper normal subgroup of $G$, and $G^{\prime}$ is the unique minimal irreducible normal subgroup of $G$. In particular, $G^{\prime}$ is perfect. In the totally imprimitive case, the countability of $\Delta$ entails that $\operatorname{dim}_{\mathbb{F}} V$ is countable, and $G$ is countable from [23, 1.19] and the theorem of A. E. Zalesskii [28] and D. J. Winter [27], applied to $V_{0}$. In modification of the usual terminology, we say that $G$ is almost primitive if it is either an almost primitive finitary linear group in the usual sense, or a primitive group of finitary permutations.

We also note that every non-trivial normal subgroup $N$ of $G$ either is irreducible, or the $N$-homogeneous components in $V$ are finite-dimensional: In the case when there is more than one $N$-homogeneous component in $V$, these components form proper $G$-blocks and must therefore be finite-dimensional. In the other case the unique $N$-homogeneous component is a direct sum of finitely many copies of an infinite-dimensional irreducible $\mathbb{F} N$-module, since $N$ acts finitarily on $V$. But $G$ acts finitarily on $V$ too, and therefore the component merely consists of one copy of the irreducible $\mathbb{F} N$-module.

2.3. Ultraproducts (see [5], Appendix). A set $\Phi$ of subsets of the set $I$ is said to be a filter on $I$ if the following conditions are satisfied:

(i) $\emptyset \notin \Phi$,

(ii) $X, Y \in \Phi$ imply $X \cap Y \in \Phi$, and

(iii) $X \subseteq Y \subseteq I$ and $X \in \Phi$ implies $Y \in \Phi$.

It is a straightforward consequence of Zorn's Lemma that every filter on $I$ is contained in an ultrafilter on $I$, that is, a filter $\Phi$ with the additional property that for every $X \subseteq I$, either $X \in \Phi$ or $I-X \in \Phi$.

Suppose now that $\left\{S_{i} \mid i \in I\right\}$ is a family of non-empty sets. Then every ultrafilter $\Phi$ on $I$ gives rise to an equivalence relation $\sim_{\Phi}$ on the cartesian product $\prod_{i \in I} S_{i}$ via

$$
\left(s_{i}\right)_{i \in I} \sim_{\Phi}\left(t_{i}\right)_{i \in I} \quad \text { if and only if } \quad\left\{i \in I \mid s_{i}=t_{i}\right\} \in \Phi .
$$

The set of equivalence classes $\left(\prod_{i \in I} S_{i}\right) / \Phi$ is called an ultraproduct. If for every $i \in I$ we are given a group $G_{i}$ of automorphisms of the vector space $V_{i}$ over the field $\mathbb{K}_{i}$, then $\left(\prod_{i \in I} G_{i}\right) / \Phi$ becomes a group of automorphisms of the vector space $\left(\prod_{i \in I} V_{i}\right) / \Phi$ over the field $\left(\prod_{i \in I} \mathbb{K}_{i}\right) / \Phi$, where all the operations in question are defined componentwise. Correspondingly, every ultraproduct $\left(\prod_{i \in I} G_{i}\right) / \Phi$ of permutation groups $G_{i} \leq \operatorname{Sym}\left(\Omega_{i}\right)$ acts componentwise on the set $\left(\prod_{i \in I} \Omega_{i}\right) / \Phi$. Moreover, there is the famous theorem of Loś [2 5.2.1] with the consequence that common properties of all factors of an ultraproduct, which can be formulated by first order sentences, are inherited by the ultraproduct itself [2, 5.2.2]. 


\section{Covers}

Proposition 3.1. Every almost primitive resp. totally imprimitive $\mathfrak{X}$-group has an $\omega$-cover of almost primitive resp. totally imprimitive $\mathfrak{X}$-subgroups.

Proof. It suffices to consider an uncountable and hence almost primitive $\mathfrak{X}$-group $G$. The primitive permutation groups $\operatorname{Alt}(\Omega)$ and $\operatorname{FSym}(\Omega)$ are obviously covered by countable subgroups of the same kind.

Now let $G$ be an almost primitive finitary permutation group. Then $G \leq$ $S_{0} \operatorname{wr}_{\Delta} \operatorname{FSym}(\Delta)$, where $S_{0}$ is finite. The wreath product acts on $\Omega=S_{0} \times \Delta$. Consider a countably infinite subgroup $X$ of $G$. Let $\Delta_{X}$ be the smallest countable subset of $\Delta$ such that $\operatorname{supp}_{\Omega} X \subseteq S_{0} \times \Delta_{X}$. Since $G$ acts highly transitively on $\Delta$, we can find for all $\lambda_{1}, \lambda_{2} \in S_{0} \times \Delta_{X}$ some $g \in G$ such that $\lambda_{1} g=\lambda_{2}$ and $\operatorname{supp}_{\Omega} g \subseteq S_{0} \times \Delta_{X}$. Moreover, for every $\pi \in \operatorname{Alt}\left(\Delta_{X}\right)$ there exists $h \in G$ such that $h$ induces $\pi$ on $\Delta_{X}$ and satisfies $\operatorname{supp}_{\Omega} h \subseteq S_{0} \times \Delta_{X}$. Together with $X$, all these elements $g$ and $h$ generate a countable subgroup of $G$, which acts almost primitively on $S_{0} \times \Delta_{X}$.

The periodic almost primitive finitary linear groups can be handled in the same way.

In the converse direction, we shall make extensive use of the following elementary observation.

Lemma 3.2. Let the $\omega$-cover $\mathfrak{C}$ of the group $G$ be the union of subsets $\mathfrak{C}_{n}(n \in \omega)$. Then one of the families $\mathfrak{C}_{n}$ forms an $\omega$-cover of $G$.

Proof. Assume that the assertion is false. Then there are countable subgroups $X_{n}$ $(n \in \omega)$ of $G$ such that no subgroup from $\mathfrak{C}_{n}$ contains $X_{n}$. But now the countable subgroup $\left\langle X_{n} \mid n \in \omega\right\rangle$ cannot be contained in any subgroup from the $\omega$-cover $\mathfrak{C}$, a contradiction.

We note an immediate consequence of Lemma 3.2 and Theorem A.

Corollary 3.3. Periodic irreducible finitary linear groups that are not totally imprimitive are countably recognizable.

Proof. Suppose that the periodic group $G$ has an $\omega$-cover $\mathfrak{C}$ of such groups. From Lemma 3.2 , we may assume that $\mathfrak{C}$ consists exclusively of groups of precisely one of the following types: linear groups, non-linear primitive groups, almost primitive groups. By Theorem A, we need to consider the first two cases only. Non-linear primitive groups have been treated in [13 Theorem A]. If every $X \in \mathfrak{C}$ is a periodic irreducible linear group, then $G$ is linear by Mal'cev's Theorem. From Lemma 3.2 we may assume that the characteristic of the underlying fields is independent of $X \in \mathfrak{C}$. Since every $X \in \mathfrak{C}$ has trivial unipotent radical, the same holds for $G$. Therefore $G$ is countable by [23, 1.19] and [28, [27]. In particular, $G \in \mathfrak{C}$ is an irreducible linear group.

\section{Almost Primitive Groups}

In this section we shall prove Theorem A.

Lemma 4.1. Every infinite primitive finitary permutation group admits just one non-trivial transitive finitary permutation representation, namely the natural one. 
Proof. Let $\operatorname{Alt}(\Delta) \leq G \leq \mathrm{FSym}(\Delta)$, and suppose that $G$ acts as a transitive finitary permutation group on the infinite set $\Omega$. Since $G^{\prime}$ is infinite and simple, we have $G^{\prime}=\operatorname{Alt}(\Omega)$. From [19, 3.3.1] or [3, Corollary 3.5], the complex vector space $V=[\mathbb{C} \Omega, G]$ is an irreducible $\mathbb{C} G$-module. Hence [3] Theorem B] yields $V \cong_{\mathbb{C} G}[\mathbb{C} \Delta, G]$. It remains to show that the action of every element $g \in G$ on $\Omega$ is determined by its action on $V$. But we can choose $\omega_{g} \in \Omega-\operatorname{supp}_{\Omega} g$. Then the action of $g$ on the $\mathbb{C}$-basis $\left\{\omega-\omega_{g} \mid \omega \in \Omega-\left\{\omega_{g}\right\}\right\}$ of $V$ is similar to the action of $g$ on $\Omega-\left\{\omega_{g}\right\}$.

Lemma 4.2. Let $V_{0}$ be a finite-dimensional $\mathbb{F}$-vector space, and let $\Delta$ be an infinite set. Consider the canonical finitary linear representation of the restricted wreath product $W=\mathrm{GL}_{\mathbb{F}}\left(V_{0}\right) \mathrm{wr}_{\Delta} \operatorname{FSym}(\Delta)$ on the vector space $V=V_{0} \otimes \Delta$. Let $G$ be a subgroup of $W$ such that the induced action of $G$ on $\Delta$ is primitive, and such that the normalizer in $G$ of $V_{0} \otimes \delta$ induces an irreducible subgroup of $\mathrm{GL}_{\mathbb{F}}\left(V_{0} \otimes \delta\right)$ for each $\delta \in \Delta$. Then $G$ acts irreducibly on $V$.

Proof. Consider a non-zero vector $v=\sum_{\delta \in \Delta_{0}} v_{\delta} \otimes \delta$ in $V$, where $\Delta_{0}$ is a finite subset of $\Delta$. Fix some $\delta_{0} \in \Delta_{0}$ with $v_{\delta_{0}} \neq 0$. Let $S$ be the normalizer in $G$ of $V_{0} \otimes \delta_{0}$, and let $S_{0}$ be a finite subset of $S$ such that $\mathbb{F} S_{0}$ and $\mathbb{F} S$ induce the same subspace of $\operatorname{End}_{\mathbb{F}}\left(V_{0} \otimes \delta_{0}\right)$ on $V_{0} \otimes \delta_{0}$. Without loss we may assume that $S_{0}$ fixes every vector in $V_{0} \otimes\left(\Delta-\Delta_{0}\right)$. Since $G$ acts highly transitively on $\Delta$, we can find $h \in G$ such that $h$ normalizes $V_{0} \otimes \delta_{0}$, and such that the permutation of $\Delta$ induced from $h$ moves $\Delta_{0}-\left\{\delta_{0}\right\}$ into $\Delta-\Delta_{0}$. But then $V_{0} \otimes \delta_{0} \leq\left[v, S_{0}^{h}\right]$, and hence $V \leq[v, G]$.

Theorem 4.3. Periodic almost primitive finitary linear groups are countably recognizable.

Proof. Suppose that the group $G$ has an $\omega$-cover $\mathfrak{C}$ of subgroups which are almost primitive finitary linear groups on some infinite-dimensional vector spaces. Each $X \in \mathfrak{C}$ is an irreducible subgroup of the restricted wreath product $W_{X}=$ $S_{X} \operatorname{wr}_{\Delta_{X}} \operatorname{FSym}\left(\Delta_{X}\right)$, where $S_{X} \leq \mathrm{GL}_{\mathbb{F}_{X}}\left(V_{X, 0}\right)$ for some finite- (possibly zero-) dimensional $\mathbb{F}_{X}$-vector space $V_{X, 0}$, and where the image of the canonical homomorphism $\pi_{X}: X \rightarrow \operatorname{FSym}\left(\Delta_{X}\right)$ contains $\operatorname{Alt}\left(\Delta_{X}\right)$. The wreath product $W_{X}$ acts naturally on the vector space $V_{X}=V_{X, 0} \otimes \Delta_{X}$, and the irreducible subgroup $S_{X}$ of $\mathrm{GL}_{\mathbb{F}_{X}}\left(V_{X, 0}\right)$ is induced from the normalizer of $V_{X, 0} \otimes \delta_{X, 0}$ in $X$, for some fixed $\delta_{X, 0} \in \Delta_{X, 0}$. Each $g \in X$ has the form $g=f_{X, g} \cdot g \pi_{X}$ for some $f_{X, g}: \Delta_{X} \rightarrow S_{X}$. From [23 1.19] we may assume that the fields $\mathbb{F}_{X}$ are algebraically closed.

For $X \in \mathfrak{C}$, let $P_{X}=\{Y \in \mathfrak{C} \mid X \leq Y\}$. Since the infinite simple group $\operatorname{Alt}\left(\Delta_{X}\right)$ contains finite $p$-subgroups of arbitrary large solubility lengths, [23, 9.20 and 9.4] imply that $\operatorname{Alt}\left(\Delta_{X}\right)$ cannot be a composition factor of the base group of $W_{Y}$ for any $Y \in P_{X}$. In particular, $K_{X, Y}=X \cap \operatorname{ker} \pi_{Y} \leq \operatorname{ker} \pi_{X}$. Let $K_{X}=\bigcap_{Y \in P_{X}} K_{X, Y}$. Note that $K_{X_{1}} \leq K_{X_{2}}$ whenever $X_{1} \leq X_{2}$. Therefore $K=\bigcup_{X \in \mathfrak{C}} K_{X}$ is a normal subgroup in $G$ with $K \cap X=K_{X}$. Let $\pi: G \rightarrow G / K$ denote the canonical epimorphism. Consider a finite subgroup $F$ of $G^{\prime}$. Choose $X \in \mathfrak{C}$ such that $F \leq X^{\prime}$ and $K \cap F=F \cap \operatorname{ker} \pi_{X}$. Let $\Xi=\operatorname{supp}_{\Delta_{X}} F \pi_{X}$. Then $F \pi_{X} \leq X^{\prime} \pi_{X}=\operatorname{Alt}\left(\Delta_{X}\right)$, and so $F$ is contained in a finite subgroup $\bar{F}$ of $X$ such that $\bar{F} \pi_{X}=\operatorname{Alt}(\Xi)$. This shows that $G^{\prime} \pi$ has a Kegel cover with alternating quotients. Hence [10, Theorem 4.1] yields that $G^{\prime} \pi$ is simple.

Next, consider a finite subgroup $F$ of $G$ with $\left(F \cap G^{\prime}\right) \pi \neq 1$. Let $\Phi$ be an ultrafilter on $\mathfrak{C}$ which contains the sets $P_{X}(X \in \mathfrak{C})$. From Lemma 3.2 we may assume 
that there exists $m \in \omega$ such that $\left|\operatorname{supp}_{\Delta_{X}} F \pi_{X}\right|=m$ for all $X \in \mathfrak{C}$. Now $G \pi \leq$ $\left(\prod_{X \in \mathfrak{C}} \operatorname{FSym}\left(\Delta_{X}\right)\right) / \Phi$ via $g \pi=\left(g \pi_{X}\right)_{X \in \mathfrak{C}}$ for all $g \in G$; here $g \pi_{X}=1$ whenever $g \notin X \in \mathfrak{C}$. The ultraproduct acts faithfully on the set $\Sigma=\left(\prod_{X \in \mathfrak{C}} \Delta_{X}\right) / \Phi$. From Łoś Theorem, $\left|\operatorname{supp}_{\Sigma} F \pi\right|=m$. Since $\operatorname{FSym}(\Sigma)$ is a normal subgroup of $\operatorname{Sym}(\Sigma)$, the simplicity of $G^{\prime} \pi$ enforces that $\left(G^{\prime} F\right) \pi \leq \mathrm{FSym}(\Sigma)$. Let $\Delta$ be an orbit of $\left(G^{\prime} F\right) \pi$ in $\Sigma$, on which $G^{\prime} \pi$ acts non-trivially. The pointwise stabilizer $Z \pi$ in $\left(G^{\prime} F\right) \pi$ of $\Delta$ is a normal subgroup, which intersects $G^{\prime} \pi$ trivially, and which must therefore commute with $G^{\prime} \pi$. Assume that $Z \pi$ contains a non-trivial element $z \pi$. Choose $X \in \mathfrak{C}$ such that $z \in X$ and $z \pi_{X} \neq 1$. Because $\left[z, X^{\prime}\right] \leq\left[z, G^{\prime}\right] \cap X \leq$ $K \cap X \leq \operatorname{ker} \pi_{X}$ we obtain $\left[z \pi_{X}, X^{\prime} \pi_{X}\right]=1$. This contradiction shows that $\left(G^{\prime} F\right) \pi$ acts faithfully on $\Delta$. However, the group $G^{\prime} \pi$ acts transitively on $\Delta$. But then $G^{\prime} \pi=\operatorname{Alt}(\Delta)$ and $\left|\left(G^{\prime} F\right) \pi: G^{\prime} \pi\right| \leq 2$. Since $F$ was an arbitrary finite subgroup of $G$, we conclude that $\left|G \pi: G^{\prime} \pi\right| \leq 2$, and that $\operatorname{Alt}(\Delta) \leq G \pi \leq \operatorname{FSym}(\Delta)$ for some orbit $\Delta$ of $G \pi$ in the ultraproduct $\Sigma$.

In the sequel we choose $F$ such that $\left(G^{\prime} F\right) \pi=G \pi$. We may assume without loss that $F \leq X$ for all $X \in \mathfrak{C}$, and that there exists $m \in \omega$ such that $\left|\operatorname{supp}_{\Delta_{X}} F \pi_{X}\right|=$ $m$ for all $X \in \mathfrak{C}$ (Lemma 3.2). From Loś Theorem, $\left|\operatorname{supp}_{\Delta} F \pi\right| \leq m$.

Consider some $X \in \mathfrak{C}$. Note that $X \pi_{X}$ is an image of $X \pi$, with kernel $K_{X, X} \pi$. Since $X^{\prime} \pi_{X}$ cannot occur as a composition factor in a subdirect product of finite groups, there exists an infinite orbit $\Gamma_{X}$ of $X \pi$ in $\Delta$. Since $X \pi_{X}$ is non-abelian, $K_{X, X} \pi$ cannot act transitively on $\Gamma_{X}$. From Lemma 4.1 the group $X \pi_{X}$ acts naturally on the set of orbits of $K_{X, X} \pi$ in $\Gamma_{X}$. Let $\nu$ be the size of such an orbit. Then

$$
\nu \cdot\left|\operatorname{supp}_{\Gamma_{X}} F \pi\right| \leq \nu \cdot m=\nu \cdot\left|\operatorname{supp}_{\Delta_{X}} F \pi_{X}\right| \leq\left|\operatorname{supp}_{\Gamma_{X}} F \pi\right| .
$$

It follows that $\nu=1$, and that $K_{X, X} \pi$ acts trivially on $\Gamma_{X}$. Now $X \pi_{X}$ acts naturally on $\Gamma_{X}$. Moreover, $\left|\operatorname{supp}_{\Gamma_{X}} F \pi\right| \leq\left|\operatorname{supp}_{\Delta_{X}} F \pi_{X}\right|$ enforces that $\Gamma_{X}$ is the unique infinite $X \pi$-orbit in $\Delta$. This allows us to identify $\Delta_{X}$ with the subset $\Gamma_{X}$. Let $\Lambda$ be a countable subset of $\Delta$. Then there is a countable subgroup $Z$ of $G$ such that $Z \pi=\operatorname{Alt}(\Lambda)$. If $X \in \mathfrak{C}$ contains $Z$, then the infinite alternating group $Z \pi$ cannot be a composition factor of the base group of $W_{X}$. Hence $Z \cap \operatorname{ker} \pi_{X}=Z \cap K$ and $\Lambda \subseteq \Delta_{X}$. In particular, $\Delta=\bigcup_{X \in \mathfrak{C}} \Delta_{X}$ and $\Delta_{X} \subseteq \Delta_{Y}$ for $X \leq Y$.

We now form the ultraproduct $S=\left(\prod_{X \in \mathfrak{C}} S_{X}\right) / \Phi$, and define a homomorphism $\tau: G \longrightarrow W=S \mathrm{Wr}_{\Delta} \operatorname{FSym}(\Delta)$ by $g \tau=f_{g} \cdot g \pi$, where $f_{g}: \Delta \rightarrow S$ is given via

$$
(\delta) f_{g}=\left((\delta) f_{X, g}\right)_{X \in \mathfrak{C}} \quad \text { for all } \delta \in \Delta ;
$$

here $(\delta) f_{X, g}=1$ whenever $g \notin X \in \mathfrak{C}$ or $\delta \notin \Delta_{X}$. The wreath product $W$ acts on $V=V_{0} \otimes \Delta$, where $V_{0}=\left(\prod_{X \in \mathfrak{C}} V_{X, 0}\right) / \Phi$ is a vector space over the field $\mathbb{F}=\left(\prod_{X \in \mathfrak{C}} \mathbb{F}_{X}\right) / \Phi$. From Lemma 3.2 we may assume that there exists $n \in \omega$ such that $\operatorname{dim}_{\mathbb{F}_{X}} V_{X, 0}=n$ for all $X \in \mathfrak{C}$. By Eoś Theorem, $\operatorname{dim}_{\mathbb{F}} V_{0}=n$ too. We now show that $\tau$ is injective.

For any finite subset $H \subseteq X \in \mathfrak{C}$ we let $\Delta_{X, H}$ denote the smallest subset of $\Delta_{X}$ such that $H$ fixes each vector in $V_{X, 0} \otimes\left(\Delta_{X}-\Delta_{X, H}\right)$. Note that $\Delta_{X, H}$ is finite. Consider a finite subset $\Lambda \subseteq \Delta_{X}$, where $X \in \mathfrak{C}$ is fixed. There exists a finite subgroup $H \leq X$ of smallest possible order such that $H \pi_{X}=\operatorname{Alt}(\Lambda)$. Then every proper normal subgroup of $H$ is contained in $\operatorname{ker} \pi_{X}$. Consequently, if $\Lambda$ has been chosen large enough, then $H$ cannot have a non-trivial linear representation of degree $\leq n\left(\left[23,9.20\right.\right.$ and 9.4]). This shows that $\Delta_{Y, H}=\operatorname{supp}_{\Delta_{Y}} H \pi_{Y} \subseteq \operatorname{supp}_{\Delta} H \pi$ for all $Y \in P_{X}$. 
Assume that there exists $1 \neq g \in X \cap \operatorname{ker} \tau$. Choose $\Lambda$ and $H$ as above such that $\Delta_{X, g} \subseteq \Lambda$ and $\Delta_{X, g} h \cap \Delta_{X, g}=\emptyset$ for some $h \in H$. Then $[g, h] \neq 1$. For every $Y \in P_{X}$, this enforces $(\delta) f_{Y, g} \neq 1$ for some $\delta \in \operatorname{supp}_{\Delta} H \pi$. By [5] Lemma B.1], there exists $\delta \in \operatorname{supp}_{\Delta} H \pi$ such that $\left\{Y \in P_{X} \mid(\delta) f_{Y, g} \neq 1\right\} \in \Phi$. But then $(\delta) f_{g} \neq 1$ and $g \tau \neq 1$. This contradiction shows that $\tau$ is an embedding.

We shall show next that $G \tau \leq \mathrm{FGL}_{\mathbb{F}}(V)$. To this end, consider $g \in G$ and $\delta \in \Delta$ with $(\delta) f_{g} \neq 1$. Choose $X \in \mathfrak{C}$ such that $g \in X$ and $\delta \in \Delta_{X, g}$. Assume that there exists $Y \in P_{X}$ satisfying $\delta \notin \Delta_{Y, g}$. Then $\delta \notin \operatorname{supp}_{\Delta} g \pi$. Choose $\Lambda \subseteq \Delta_{X}$ and $H \leq X$ as above such that $\Lambda \cap\left(\Delta_{X, g} \cup \Delta_{Y, g}\right)=\{\delta\}$. Since $\operatorname{Alt}\left(\Delta_{Y}\right) \leq$ $Y \pi_{Y}$, there exists $t \in Y$ such that $\Delta_{X} \cap \operatorname{supp}_{\Delta} t \pi=\emptyset$ and $\Delta_{Y, H}(t \pi) \cap \Delta_{Y, g}=\emptyset$. From the action of $\left\langle g, H^{t}\right\rangle \tau$ on $V_{0} \otimes \Delta_{X}$ we see that $\left[g, H^{t}\right] \neq 1$. On the other hand, the subgroup $\left\langle g, H^{t}\right\rangle$ of $Y$ satisfies $\left[g, H^{t}\right]=1$ from its action on $V_{Y}$. This contradiction shows that $\delta \in \Delta_{Y, g}$ for every $Y \in P_{X}$. Since the join of every countable subfamily of the $\omega$-cover $\mathfrak{C}$ is contained in a subgroup from $\mathfrak{C}$, we can now find for every countable subset $\Gamma_{0}$ of $\Gamma=\left\{\delta \in \Delta \mid(\delta) f_{g} \neq 1\right\}$ some $X \in \mathfrak{C}$ such that $g \in X$ and $\Gamma_{0} \subseteq \Delta_{X, g}$. Since $\Delta_{X, g}$ is finite, $\Gamma$ must be finite too, that is, $g \tau \in \mathrm{FGL}_{\mathbb{F}}(V)$. In particular, $G \tau$ is contained in the restricted wreath product $S \mathrm{wr}_{\Delta} \operatorname{FSym}(\Delta)$.

The ultraproduct $\mathbb{F}$ of the algebraically closed fields $\mathbb{F}_{X}$ is algebraically closed too. By [23, 1.17], each $S_{X}$ contains precisely $n^{2}$ elements which are linearly independent over $\mathbb{F}_{X}$. By Łoś Theorem, $S$ too contains precisely $n^{2}$ elements which are linearly independent over $\mathbb{F}$. Hence $S$ is an irreducible subgroup of $\mathrm{GL}_{\mathbb{F}}\left(V_{0}\right)$ by [23, 1.18]. Moreover, the $n^{2}$ independent elements in $S$ are still induced from the normalizer in $G \tau$ of $V_{0} \otimes \delta_{0}$ for some fixed $\delta_{0}$. Now Lemma 4.2 ensures that $G \tau$ acts irreducibly on $V$. Since $G$ contains an infinite alternating section, $G \tau$ must act almost primitively on $V$.

Theorem 4.4. Almost primitive finitary permutation groups are countably recognizable.

Proof. Suppose that the group $G$ has an $\omega$-cover $\mathfrak{C}$ of subgroups which are almost primitive finitary permutation groups on some infinite sets. Each $X \in \mathfrak{C}$ is a transitive subgroup of the restricted wreath product $W_{X}=S_{X} \operatorname{wr}_{\Delta_{X}} \operatorname{FSym}\left(\Delta_{X}\right)$, acting on $\Omega_{X}=S_{X} \times \Delta_{X}$. Here the finite (possibly trivial) group $S_{X}$ is induced from the setwise stabilizer in $G$ of the block $S_{X} \times\left\{\delta_{X}\right\}$, for some fixed $\delta_{X} \in \Delta_{X}$. Moreover, the image of the canonical homomorphism $\pi_{X}: X \rightarrow \operatorname{FSym}\left(\Delta_{X}\right)$ contains $\operatorname{Alt}\left(\Delta_{X}\right)$. From Lemma 3.2 we may assume that the groups $S_{X}(X \in \mathfrak{C})$ are all isomorphic. We consider $S_{X}$ as a linear group. Then we can go through the proof of Theorem 4.3. Finally we obtain an embedding $\tau: G \longrightarrow W=$ $S \mathrm{wr}_{\Delta} \operatorname{FSym}(\Delta)$. This wreath product acts as a finitary permutation group on $\Omega=S \times \Delta$. And the ultraproduct $S=\left(\prod_{X \in \mathfrak{C}} S_{X}\right) / \Phi$ is induced from the setwise stabilizer in $G$ of the block $S \times\{\delta\}$, for some fixed $\delta \in \Delta$. Moreover, the image of the canonical homomorphism $\pi: G \rightarrow \operatorname{FSym}(\Delta)$ contains $\operatorname{Alt}(\Delta)$. It is easy to see that $G$ acts transitively on $\Omega$. Since $G$ contains an infinite alternating section, $G$ must act almost primitively on $\Omega$.

\section{Counterexamples}

In this section we shall construct uncountable groups with an $\omega$-cover of totally imprimitive $\mathfrak{X}$-subgroups. These so-called counterexamples will provide a proof of 
Theorem B. K. Hickin and R. Phillips have shown in [7] that totally imprimitive finitary permutation groups $G$ with the property $\left|N G^{\prime} / G^{\prime}\right|<\infty$ for every intransitive normal subgroup $N$ are countably recognizable. However they were not able to decide whether every totally imprimitive finitary permutation group $G$ has this property. The cornerstone of our construction will be examples of totally imprimitive finitary permutation groups $G$ in which this property fails, and which are therefore of independent interest.

Definition. In the sequel, a totally imprimitive finitary permutation group $G$ will be called slim if it contains an intransitive normal subgroup $N$ such that $N G^{\prime} / G^{\prime}$ is infinite.

5.1. Construction of Slim Groups. Let $\mathbb{F}_{p}$ denote the prime field in characteristic $p>0$, and let $\omega$ denote the first infinite ordinal. Choose non-trivial finite groups $S_{n}(n \in \omega)$ and non-trivial faithful irreducible $\mathbb{F}_{p} S_{n}$-modules $C_{n}(n \in \omega)$. Let $S_{n}$ act on the $\mathbb{F}_{p}$-vector space $B_{n}=\left\langle b_{n}\right\rangle \oplus C_{n}$ via $\left[b_{n}, S_{n}\right]=0$. Let $X_{n}$ be the group generated by $S_{n}$ and the stability group of the chain $0<C_{n}<B_{n}$. Let $X_{n}$ act on the $\mathbb{F}_{p}$-vector space $A_{n}=\left\langle a_{n}\right\rangle \oplus B_{n}$ via $\left[a_{n}, X_{n}\right]=0$. The group ring $\mathbb{F}_{p} X_{n}$ contains a projective indecomposable $\mathbb{F}_{p} X_{n}$-module $P_{n}$ such that head and socle of $P_{n}$ are isomorphic to $C_{n}$ ([8, VII.10.3/10.9/11.6]). Since $P_{n}$ is also an injective $\mathbb{F}_{p} X_{n}$-module ([8, VII.7.8]), and since $B_{n}$ is indecomposable, there exists an embedding $\varphi_{n}: B_{n} \rightarrow P_{n}$. Since $C_{n}$ is a non-trivial $\mathbb{F}_{p} X_{n}$-module, we can extend $\varphi_{n}$ to an embedding $\varphi_{n}: A_{n} \rightarrow \mathbb{F}_{p} X_{n}$ via $a_{n} \varphi_{n}=\sum_{x \in X_{n}} x$. Note that $A_{n} \varphi_{n}$ is contained in the augmentation ideal $\kappa\left(\mathbb{F}_{p} X_{n}\right)$. In the sequel we shall suppress $\varphi_{n}$ and identify $A_{n}$ with its image in $\kappa\left(\mathbb{F}_{p} X_{n}\right)$.

Next, let $W_{0}=X_{0}$ and $W_{n}=W_{n-1}$ wr $X_{n}$ for all $n \geq 1$. Then $W_{m}=$ $W_{n-1}$ wr $Q_{n, m}$ for all $m \geq n$, where $Q_{n, m}=X_{n}$ wr $\cdots$ wr $X_{m}$. We also let $W=$ $\bigcup_{n \in \omega} W_{n}$ and $W=W_{n-1} \operatorname{wr} Q_{n}$ for $Q_{n}=\bigcup_{m \geq n} Q_{n, m}$. We shall now recursively construct $\mathbb{F}_{p} W_{n}$-submodules $\bar{B}_{n} \leq \bar{A}_{n}$ of the base group of $C_{p}$ wr $W_{n}$ and an $\mathbb{F}_{p} X_{n}$-monomorphism $\psi_{n}: A_{n} \rightarrow \bar{A}_{n}$ such that $B_{n} \psi_{n} \leq \bar{B}_{n}$ and $\bar{A}_{n}=$ $\bar{B}_{n} \oplus\left\langle a_{n} \psi_{n}\right\rangle$ and $\left[\bar{A}_{n}, W_{n}\right] \leq \bar{B}_{n}$.

For $n=0$ we choose $\bar{B}_{0}=B_{0} \leq A_{0}=\bar{A}_{0}$ and $\psi_{0}=i d$. Because $A_{0}$ is contained in the permutation module $\mathbb{F}_{p} X_{0}$, the semidirect product $B_{0} \rtimes W_{0}$ can be viewed as a transitive subgroup of $C_{p}$ wr $W_{0}$. Suppose next that $\bar{A}_{n}, \bar{B}_{n}, \psi_{n}$ have been constructed for some $n$. Consider the action of $W_{n+1}=W_{n}$ wr $X_{n+1}$ on $\bar{A}_{n}^{X_{n+1}}=\bigoplus_{x \in X_{n+1}} \bar{A}_{n}^{x} \leq C_{p}$ wr $W_{n+1}$. Let $K_{n}$ be the kernel of the trace map

$$
\bar{A}_{n}^{X_{n+1}} \ni\left(a_{x}\right)_{x \in X_{n+1}} \longmapsto \sum_{x \in X_{n+1}} a_{x} \in \bar{A}_{n}
$$

Clearly $\left\langle a_{n} \psi_{n}\right\rangle^{X_{n+1}} \cong \mathbb{F}_{p} X_{n+1}$ and $K_{n} \cap\left\langle a_{n} \psi_{n}\right\rangle^{X_{n+1}} \cong \kappa\left(\mathbb{F}_{p} X_{n+1}\right)$ as $\mathbb{F}_{p} X_{n+1^{-}}$ modules. We can therefore find an $\mathbb{F}_{p} X_{n+1}$-monomorphism $\psi_{n+1}: A_{n+1} \rightarrow K_{n} \cap$ $\left\langle a_{n} \psi_{n}\right\rangle^{X_{n+1}}$ and choose

$$
\bar{B}_{n+1}=\bar{B}_{n}^{X_{n+1}} \oplus B_{n+1} \psi_{n+1} \leq \bar{B}_{n}^{X_{n+1}} \oplus A_{n+1} \psi_{n+1}=\bar{A}_{n+1} .
$$

It remains to show that $\bar{B}_{n+1}$ and $\bar{A}_{n+1}$ are $W_{n+1}$-invariant. Clearly they are $X_{n+1^{-}}$ invariant. Moreover $\left[A_{n+1} \psi_{n+1}, W_{n}\right] \leq\left[\left\langle a_{n} \psi_{n}\right\rangle, W_{n}\right] \leq\left[\bar{A}_{n}, W_{n}\right] \leq \bar{B}_{n}$, whence $\left[\bar{A}_{n+1}, W_{n}\right] \leq \bar{B}_{n} \leq \bar{B}_{n+1}$. This completes the recursion.

Now $B=\bigcup_{n \in \omega} \bar{B}_{n}$ is an $\mathbb{F}_{p} W$-submodule of the base group of $C_{p}$ wr $W$ such that $G=B \rtimes W$ is a totally imprimitive subgroup of $C_{p}$ wr $W$. In the next section 
we shall show that $B G^{\prime} / G^{\prime}$ is infinite, which of course means that the group $G$ is slim. In the sequel we shall suppress $\psi_{n}$ and identify $A_{n}$ with its image in $\bar{A}_{n}$.

\subsection{Additional Properties of the Group $G$.}

5.2.1. $\left[a_{n}, W_{n}\right]=0$ for all $n$.

Proof. Clearly $\left[a_{0}, W_{0}\right]=\left[a_{0}, X_{0}\right]=0$. And if $\left[a_{n}, W_{n}\right]=0$, then $a_{n+1} \in A_{n+1} \leq$ $\left\langle a_{n}\right\rangle^{X_{n+1}}$ is centralized by $W_{n}^{X_{n+1}}$. On the other hand, $\left[a_{n+1}, X_{n+1}\right]=0$ by choice of $a_{n+1}$. Altogether, $\left[a_{n+1}, W_{n+1}\right]=0$.

This implies

5.2.2. $\left[B_{n+1}, W_{n}^{W}\right] \leq\left[\left\langle a_{n}\right\rangle^{X_{n+1}}, W_{n}^{X_{n+1}}\right]=0$,

and so

5.2.3. $\left[\bar{B}_{n+1}, W_{n+1}\right]=\left(\left[\bar{B}_{n}, W_{n}\right]^{X_{n+1}}+\left(K_{n} \cap \bar{B}_{n}^{X_{n+1}}\right)\right) \oplus\left[B_{n+1}, X_{n+1}\right]$.

In particular,

5.2.4. $\left[\bar{B}_{n+1}, W_{n+1}\right] \cap \bar{B}_{n}=\left[\bar{B}_{n}, W_{n}\right]$ and $[B, W]=\bigcup_{n \in \omega}\left[\bar{B}_{n}, W_{n}\right]$.

5.2.5. $G$ is a slim group.

Proof. Since $B G^{\prime} / G^{\prime} \cong B / B \cap G^{\prime} \cong B /[B, W]$, it suffices to show that $p^{n+1}=$ $\left|\bar{B}_{n} /\left[\bar{B}_{n}, W_{n}\right]\right|$ for all $n$. But $\left|\bar{B}_{0} /\left[\bar{B}_{0}, W_{0}\right]\right|=p$, and induction yields

$$
\begin{aligned}
\left|\bar{B}_{n+1} /\left[\bar{B}_{n+1}, W_{n+1}\right]\right| & =\left|\bar{B}_{n}^{X_{n+1}} /\left(\left[\bar{B}_{n}, W_{n}\right]^{X_{n+1}}+\left(K_{n} \cap \bar{B}_{n}^{X_{n+1}}\right)\right)\right| \cdot\left|B_{n+1} / C_{n+1}\right| \\
& =\left|\bar{B}_{n} /\left[\bar{B}_{n}, W_{n}\right]\right| \cdot p=p^{n+2} .
\end{aligned}
$$

5.2.6. $C_{B}\left(W_{n}^{W}\right)=\bigoplus_{k \geq 1} B_{n+k}^{Q_{n+k+1}}$ for all $n$.

Proof. It suffices to calculate that $C_{\bar{B}_{m}}\left(W_{n}^{W}\right)=B_{n+1}^{Q_{n+2, m}} \oplus \cdots \oplus B_{m}^{Q_{m+1, m}}$ for $m \geq n$ (where $Q_{k, \ell}=1$ for $k>\ell$ ). Consider the case $n=m$ first. Clearly $C_{\bar{B}_{0}}\left(W_{0}^{W}\right)=$ $C_{\bar{B}_{0}}\left(W_{0}\right)=0$. And if $C_{\bar{B}_{n}}\left(W_{n}^{W}\right)=0$, then $C_{\bar{B}_{n}^{X_{n+1}}}\left(W_{n+1}^{W}\right) \leq C_{\bar{B}_{n}^{X_{n+1}}}\left(W_{n}^{W}\right)=0$, whence

$$
\begin{aligned}
C_{\bar{B}_{n+1}}\left(W_{n+1}^{W}\right) & \cong C_{\bar{B}_{n+1}}\left(W_{n+1}^{W}\right) \bar{B}_{n}^{X_{n+1}} / \bar{B}_{n}^{X_{n+1}} \\
& \leq C_{\bar{B}_{n+1} / \bar{B}_{n}^{X+1}}\left(X_{n+1}\right) \cong C_{B_{n+1}}\left(X_{n+1}\right)=0 .
\end{aligned}
$$

Suppose now that $C_{\bar{B}_{m}}\left(W_{n}^{W}\right)$ has the desired form for some $m \geq n$. Then 5.2.2 gives

$$
\begin{aligned}
C_{\bar{B}_{m+1}}\left(W_{n}^{W}\right) & =\left(C_{\bar{B}_{m}}\left(W_{n}^{W}\right)\right)^{X_{m+1}} \oplus B_{m+1} \\
& =\left(B_{n+1}^{Q_{n+2, m}} \oplus \cdots \oplus B_{m}^{Q_{m+1, m}}\right)^{X_{m+1}} \oplus B_{m+1}^{Q_{m+2, m+1}} \\
& =B_{n+1}^{Q_{n+2, m+1}} \oplus \cdots \oplus B_{m+1}^{Q_{m+2, m+1}} .
\end{aligned}
$$


It is evident now that the centralizers $C_{B}\left(W_{n}^{W}\right)(n \in \omega)$ form a strictly descending chain in $B$. Since $G$ is finitary, their intersection is trivial. Let $C$ denote the completion of $B$ with respect to the residual system $\left\{C_{B}\left(W_{n}^{W}\right) \mid n \in \omega\right\}$. Let $C_{0}$ be the set of all $c \in C$ satisfying

$$
c \in \bigcap_{m \geq n}\left(\bar{B}_{n} \oplus B_{n+1} \oplus \cdots \oplus B_{m} \oplus C_{C}\left(W_{m}^{W}\right)\right) \quad \text { for some } n=n(c) \in \omega .
$$

Obviously $C_{0}$ is a group of cardinality $2^{\aleph_{0}}$.

5.2.7. $C_{0}$ is an $\mathbb{F}_{p} W$-module.

Proof. If $c \in C_{0}$ and $w \in W$, then there exists $n \in \omega$ such that $w \in W_{n}$ and $c=$ $b_{m}+d_{m}$ for all $m \geq n$, where $b_{m} \in \bar{B}_{n}=\bar{B}_{n}^{w}$ and $d_{m} \in B_{n+1}+\cdots+B_{m}+$ $C_{C}\left(W_{m}^{W}\right) \leq C_{C}\left(W_{n}^{W}\right) \leq C_{C}(w)$. Then $\left(b_{m}+d_{m}\right)^{w} \in \bar{B}_{n}+B_{n+1}+\cdots+B_{m}+$ $C_{C}\left(W_{m}^{W}\right)$ for all $m \geq n$, and so $c^{w}=\bigcap_{m \geq n}\left(b_{m}+d_{m}\right)^{w}+C_{C}\left(W_{m}^{W}\right) \in C_{0}$.

It follows from 5.2.6 that

5.2.8. $\bigcap_{n \in \omega}\left([B, W]+C_{B}\left(W_{n}^{W}\right)\right)=\bigcap_{n \in \omega}\left([B, W] \oplus \bigoplus_{k \geq 1}\left\langle b_{n+k}\right\rangle\right)=[B, W]$.

This also implies

5.2.9. $[B, W]=\bigcap_{n \in \omega}\left([B, W]+C_{C}\left(W_{n}^{W}\right)\right)$.

Remark 5.2.10. We can choose for $S_{n}$ any finite simple group $\neq C_{p}$. Then $G$ has its composition factors isomorphic to $C_{p}$ and $S_{n}(n \in \omega)$. In particular, choosing all $S_{n}$ cyclic of prime order $q \neq p$ yields a locally solvable $\{p, q\}$-group $G$. One can also obtain slim $p$-groups by choosing all $X_{n}$ elementary abelian of order $p^{2}$, and replacing $A_{n}$ by the last but one non-trivial term of the Loewy series of $\mathbb{F}_{p} X_{n}$. Slim $p$-groups will however not lead to counterexamples as in Section 5.3, since totally imprimitive $p$-groups are countably recognizable by Theorem D (the centralizers $C_{B}\left(W_{n}^{W}\right)$ behave differently).

5.3. Construction of Counterexamples. We shall show, in this section, that the uncountable group $C_{0} \rtimes W$ has an $\omega$-cover of subgroups which are isomorphic to totally imprimitive subgroups of the wreath product $C_{p}$ wr $W$ containing $W$. Let $\Omega$ be the set on which $W$ acts canonically. By [19, 3.3.3] the group $W$ acts irreducibly on $\mathbb{C} \Omega$. Since $C_{p}$ has a non-trivial one-dimensional complex representation, every subgroup of $C_{p}$ wr $W$ containing $W$ has a faithful irreducible finitary linear representation on $\mathbb{C} \Omega$ too. Therefore $C_{0} \rtimes W$ will be a counterexample which proves Theorem B.

Consider a countable subgroup $H$ of $C_{0} \rtimes W$ containing $G=B \rtimes W$. Then $\left[C_{0}, W\right]=[B, W] \leq H$. We choose $H$ such that $\left|H \cap C_{0}:[B, W]\right|$ is infinite; that is, $H \cap C_{0}=[B, W] \oplus E$, where $E=\bigoplus_{i>1}\left\langle c_{i}\right\rangle$. It suffices to embed such a group $H$ onto a transitive subgroup of $C_{p}$ wr $W$.

Let $\Omega_{n}$ be the non-trivial orbit of $W_{n}$ in $\Omega$. We shall recursively construct embeddings $\alpha_{k}: H \longrightarrow C_{p} \mathrm{Wr}_{\Omega} W(k \geq 0)$ such that the following hold:

- $\left.\alpha_{k}\right|_{W}=i d_{W}$, and $\left(H \cap C_{0}\right) \alpha_{k}$ lies in the base group of $C_{p} \operatorname{Wr}_{\Omega} W$;

- for every $c \in H \cap C_{0}$ there exists $n \in \omega$ such that the base group function $c \alpha_{k}: \Omega \rightarrow C_{p}$ is constant on each of the orbits of the pointwise stabilizer of $\Omega_{n}$ in $W$;

$-c_{j} \alpha_{k}=c_{j} \alpha_{k-1}$ for all $j<k$, and $c_{k} \alpha_{k}$ is finitary (note that $c_{1} \alpha_{k}, \ldots, c_{k} \alpha_{k}$ are finitary then). 
Let us begin with the construction of $\alpha_{0}$. Let $\Psi$ be an ultrafilter on $\omega$ containing all cofinite subsets of $\omega$. By Łoś Theorem, $\left(\prod_{m \in \omega} C_{p}\right) / \Psi \cong C_{p}$. Therefore a $W$-homomorphism

$$
\alpha_{0}: C_{0} \longrightarrow\left(\left(\prod_{m \in \omega} C_{p}\right) / \Psi\right) \mathrm{Wr}_{\Omega} W=C_{p} \mathrm{Wr}_{\Omega} W
$$

is given via

$$
\begin{array}{ll}
(\nu)\left(c \alpha_{0}\right)=\left((\nu) d_{m}\right)_{m \in \omega} & \text { for all } \nu \in \Omega, \text { whenever } \\
c=\bigcap_{m \geq n}\left(d_{m}+C_{C}\left(W_{m}^{W}\right)\right) \quad \text { with } \quad d_{m} \in \bar{B}_{n}+B_{n+1}+\cdots+B_{m}
\end{array}
$$

$\alpha_{0}$ is injective: If $c \in \operatorname{ker} \alpha_{0}$, then $\left\{m \in \omega \mid m \geq n\right.$ and $\left.\left(\Omega_{\ell}\right) d_{m}=0\right\} \in \Psi$ for all $\ell \in \omega$, whence $c \in \bigcap_{\ell \in \omega} C_{C}\left(W_{\ell}^{W}\right)=0$. Thus $\alpha_{0}$ gives rise to an embedding $\alpha_{0}: H \longrightarrow C_{p} \operatorname{Wr}_{\Omega} W$ via $\left.\alpha_{0}\right|_{W}=i d_{W}$. The second of the above requirements for $\alpha_{0}$ is satisfied by choice of $C_{0}$.

Suppose now that $\alpha_{k}$ has been constructed for some $k$. We shall provide $\alpha_{k+1}$. Choose $n=n(k)$ such that

(a) $\operatorname{supp} c_{j} \alpha_{k} \subseteq C_{p} \times \Omega_{n}$ for all $j \leq k$,

(b) $c_{k+1} \alpha_{k}$ is constant on the orbits of the pointwise stabilizer of $\Omega_{n}$ in $W$, and

(c) $c_{1}, \ldots, c_{k+1}$ are independent modulo $[B, W]+C_{H \cap C_{0}}\left(W_{n}^{W}\right)$ (this condition can be realized because of 5.2.9 above).

Let $U$ be the setwise stabilizer of $\Omega_{n}$ in $W$, let $T$ be a right transversal of $U$ in $W$ containing 1 , and let $T_{0} \subseteq T$ be a set of representatives of the double cosets $U w U(w \in W)$ containing 1 .

Lemma 5.3.1. The elements $t\left(c_{j} \alpha_{k}\right) t^{-1}\left(t \in T_{0}, j \leq k+1\right)$ are independent modulo $\left([B, U]+C_{H \cap C_{0}}\left(W_{n}^{W}\right)\right) \alpha_{k}$.

Proof. Suppose that

$$
\sum_{t \in T_{0}} \sum_{j \leq k+1} \lambda_{t, j} t\left(c_{j} \alpha_{k}\right) t^{-1} \equiv 0 \quad \text { modulo }\left([B, U]+C_{H \cap C_{0}}\left(W_{n}^{W}\right)\right) \alpha_{k}
$$

for certain $\lambda_{t, j} \in \mathbb{F}_{p}$. Since we calculate modulo $C\left(W_{n}^{W}\right)$, condition (b) allows us to assume that $\operatorname{supp} c_{k+1} \alpha_{k} \subseteq C_{p} \times \Omega_{n}$, and hence $\operatorname{supp} t\left(c_{j} \alpha_{k}\right) t^{-1} \subseteq C_{p} \times \Omega_{n} t^{-1}$ for all $t \in T_{0}, j \leq k+1$. Since $T_{0}$ represents the double cosets $U w U(w \in W)$, the sets $\Omega_{n} t_{1}^{-1}$ and $\Omega_{n} t_{2}^{-1}$ lie in different $U$-orbits for $t_{1} \neq t_{2}$ from $T_{0}$. Hence

$\sum_{j \leq k+1} \lambda_{t, j} t\left(c_{j} \alpha_{k}\right) t^{-1} \equiv 0 \quad$ modulo $\left([B, U]+C_{H \cap C_{0}}\left(W_{n}^{W}\right)\right) \alpha_{k} \quad$ for every $t \in T_{0}$.

Conjugation with $t$ and application of $\alpha_{k}^{-1}$ yields

$$
\sum_{j \leq k+1} \lambda_{t, j} c_{j} \equiv 0 \quad \text { modulo }[B, W]+C_{H \cap C_{0}}\left(W_{n}^{W}\right) \quad \text { for every } t \in T_{0}
$$

And condition (c) implies $\lambda_{t, j}=0$ for all $t \in T_{0}, j \leq k+1$. 
Let $D$ be the group of all constant functions $\Omega_{n} \rightarrow C_{p}$. Because of Lemma 5.3.1 we can find a homomorphism $\varphi_{k}:\left(H \cap C_{0}\right) \alpha_{k} \cdot U \longrightarrow D$ such that

(i) $\left([B, U]+C_{H \cap C_{0}}\left(W_{n}^{W}\right)\right) \alpha_{k} \varphi_{k}=0$,

(ii) $c_{j} \alpha_{k} \varphi_{k}=0$ for $j \leq k+1$,

(iii) $\left(t\left(c_{j} \alpha_{k}\right) t^{-1}\right) \varphi_{k}=\left.t\left(c_{j} \alpha_{k}\right)^{-1} t^{-1}\right|_{\Omega_{n}}$ for $1 \neq t \in T_{0}, j \leq k+1$, and

(iv) $U \varphi_{k}=0$.

The choice in (iv) is possible, since $\left(H \cap C_{0}\right) \alpha_{k} \cdot U \equiv\left(H \cap C_{0}\right) \alpha_{k} \times U$ modulo $[B, U] \alpha_{k}$. A derivation $\delta_{k}$ of $H \alpha_{k}$ into the base group of $C_{p} \mathrm{Wr}_{\Omega} W$ is now given via $W \delta_{k}=0$ and

$$
\left.c \alpha_{k} \delta_{k}\right|_{\Omega_{n} \tau}=\tau^{-1}\left(\tau\left(c \alpha_{k}\right) \tau^{-1}\right) \varphi_{k} \tau \quad \text { for all } c \in H \cap C_{0} \text { and } \tau \in T .
$$

We define the group homomorphism $\alpha_{k+1}: H \longrightarrow C_{p} \mathrm{Wr}_{\Omega} W$ via $h \alpha_{k+1}=$ $h \alpha_{k} \cdot h \alpha_{k} \delta_{k}$ for all $h \in H$. We show that $\alpha_{k+1}$ is an embedding: Suppose that $h \in \operatorname{ker} \alpha_{k+1}$. Then $h \in H \cap C_{0}$, and $h \alpha_{k}=-\left(h \alpha_{k} \delta_{k}\right)$ is constant on each of the blocks $\Omega_{n} \tau(\tau \in T)$. It follows that $h \alpha_{k} \in\left(C_{H \cap C_{0}}\left(W_{n}^{W}\right)\right) \alpha_{k} \unlhd H \alpha_{k}$. But then $\tau\left(h \alpha_{k}\right) \tau^{-1} \in \operatorname{ker} \varphi_{k}$ for all $\tau \in T$, whence $h \alpha_{k}=-\left(h \alpha_{k} \delta_{k}\right)=0$. Thus, $\alpha_{k+1}$ is injective.

For every $1 \neq \tau \in T$ there is a unique $1 \neq t \in T_{0}$ such that $\tau U=t U$. Then

$$
\tau\left(c_{j} \alpha_{k}\right) \tau^{-1} \equiv t\left(c_{j} \alpha_{k}\right) t^{-1} \quad \text { modulo }[B, U] \alpha_{k} \leq \operatorname{ker} \varphi_{k} \quad \text { for all } j \leq k+1 .
$$

Moreover, (a) and (b) imply that $c_{j} \alpha_{k}$ is constant on $\Omega_{n} t U$, whence

$$
\begin{aligned}
\left(\tau\left(c_{j} \alpha_{k}\right) \tau^{-1}\right) \varphi_{k} & =\left(t\left(c_{j} \alpha_{k}\right) t^{-1}\right) \varphi_{k} \\
& =\left.t\left(c_{j} \alpha_{k}\right)^{-1} t^{-1}\right|_{\Omega_{n}}=\left.\tau\left(c_{j} \alpha_{k}\right)^{-1} \tau^{-1}\right|_{\Omega_{n}}
\end{aligned}
$$

for all $j \leq k+1$. It follows that $c_{j} \alpha_{k+1}=c_{j} \alpha_{k}$ for $j \leq k$, and that $\operatorname{supp} c_{k+1} \alpha_{k+1}$ $\subseteq C_{p} \times \Omega_{n}$. Moreover, the definition of $\delta_{k}$ and the corresponding property of $\alpha_{k}$ imply directly that for every $c \in H \cap C_{0}$ there exists $m \geq n$ such that $c \alpha_{k+1}$ is constant on each of the orbits of the pointwise stabilizer of $\Omega_{m}$ in $W$. The construction of $\alpha_{k+1}$ is complete.

Finally, we choose the ultrafilter $\Psi$ on $\omega$ as above and define a homomorphism

$$
\alpha: H \longrightarrow\left(\left(\prod_{k \in \omega} C_{p}\right) / \Psi\right) \mathrm{Wr}_{\Omega} W=C_{p} \mathrm{Wr}_{\Omega} W
$$

diagonally; that is, $\left.\alpha\right|_{W}=i d_{W}$ and

$$
(\nu)(c \alpha)=\left((\nu)\left(c \alpha_{k}\right)\right)_{k \in \omega} \quad \text { for all } c \in H \cap C_{0} \text { and } \nu \in \Omega .
$$

Here $E \alpha$ is finitary, $\operatorname{since} \operatorname{supp} c_{k} \alpha=\operatorname{supp} c_{k} \alpha_{k}$ for all $k$. And $[B, W] \alpha$ is finitary too, since $\operatorname{supp}[b, w] \alpha_{k} \subseteq C_{p} \times \operatorname{supp}_{\Omega} w$ for all $b \in B, w \in W, k \in \omega$. It follows that $H \alpha$ is a totally imprimitive subgroup of the restricted wreath product $C_{p} \operatorname{wr}_{\Omega} W$.

It remains to show that $\alpha$ is injective. Clearly $\operatorname{ker} \alpha \leq H \cap C_{0}$. Assume that $1 \neq c \in \operatorname{ker} \alpha$. Then $1 \neq[c, w] \in[B, W] \cap \operatorname{ker} \alpha$ for some $w \in W$. But $\operatorname{supp}[c, w] \alpha_{k}$ is uniformly bounded for all $k \in \omega$, whence $[c, w] \alpha \neq 1$ (see [5, Lemma B.1]). Thus $\alpha$ is the desired embedding. 
5.4. Finitary Linear Representations of the Counterexamples. Although the counterexample $Z=C_{0} \rtimes W \cong\left(C_{0} \rtimes W\right) \alpha_{0} \leq C_{p} \mathrm{Wr}_{\Omega} W$ is not irreducible finitary linear, we note that the wreath product has a faithful finitary linear representation on the $\mathbb{F}_{p}$-vector space with basis $\Omega \cup\{x\}$ via $\left[x, C_{p} \operatorname{Wr}_{\Omega} W\right]=0$ and

$$
(\nu)(f w)=((\nu) f) \cdot x+(\nu) w \quad \text { for all } \nu \in \Omega, w \in W \text { and } f: \Omega \rightarrow C_{p}=\mathbb{F}_{p}^{+} .
$$

However, $Z$ has no faithful finitary linear representation over any field of characteristic $q \geq 0$ coprime to $p$ : Otherwise the non-existence of a unipotent normal subgroup in $Z$ would show, that $Z$ is a subdirect product of irreducible finitary linear groups $Z_{i}(i \in I)$ over a field of characteristic $q$ ([19, Proposition 1]). Since $Z$ does not contain an infinite simple section, the groups $Z_{i}$ are totally imprimitive and countable. Now $Z^{\prime}$ is a countable subgroup of the uncountable group $Z$. Therefore there exists a countable subset $J \subseteq I$ such that each group $Z_{i}(i \in I \backslash J)$ is abelian. Let $K$ denote the kernel of the canonical projection of $Z$ into $\prod_{j \in J} Z_{j}$. Then $\left[K, Z^{\prime}\right] \leq K \cap Z^{\prime}=1$, whence $1 \neq K \leq C_{Z}\left(Z^{\prime}\right)=1$. This contradiction shows that faithful finitary linear representations of $Z$ are just possible over fields of characteristic $p$. But then the direct product $Z \times \operatorname{Tor}\left(\prod_{n \in \omega} C_{p^{n}}\right)$ is a group which has no faithful finitary linear representation at all, although its countable subgroups are finitary permutation groups (cf. [11, Example 3.4]).

\section{Totally Imprimitive Groups}

This section is devoted to the proof of Theorem C. In the sequel, we will use the following standard notation for iterated commutators: $[a, 0, b]=a$ and $\left[a,,_{k+1} b\right]=$ $[[a, k b], b]$ for all $k \in \omega$.

Lemma 6.1. Every finite group satisfies the identity $\left[x_{\ell} y\right]=\left[x_{\ell-k} y\right]$ for suitable positive integers $k<\ell$.

Proof. Let $S$ be a finite group. For all $a, b \in S$, there exist positive integers $k_{a, b}<\ell_{a, b}$ such that $\left[a,_{m} b\right]=\left[a,_{m-k_{a, b}} b\right]$ for all $m \geq \ell_{a, b}$. Then $S$ satisfies the required identity with $k=\prod_{a, b \in S} k_{a, b}$ and $\ell=\prod_{a, b \in S} \ell_{a, b}$.

Lemma 6.2. Let $S=\langle g, h\rangle$ be a group such that the conjugates $h^{-j} g h^{j}$ commute for $0 \leq j \leq \ell$. Then $[g, \ell h] \cdot[g, \ell-k, h]^{-1} \equiv g^{h^{\ell}} \bmod \left\langle g, g^{h}, \ldots, g^{h^{\ell-1}}\right\rangle$ for $0 \leq k<\ell$.

Proof. Induction shows that $\left[g,{ }_{j} h\right] \equiv g^{h^{j}} \bmod \left\langle g, g^{h}, \ldots, g^{h^{(j-1)}}\right\rangle$ for $0 \leq j \leq \ell$.

As a first step towards Theorem $\mathrm{C}$ we establish a counterpart to [7, Lemma 1] (see Proposition 6.6) for totally imprimitive finitary linear groups.

Proposition 6.3. Let the periodic group $G$ have an $\omega$-cover of totally imprimitive finitary linear groups. Then the commutator subgroup $G^{\prime}$ of $G$ is countable. In particular, $G$ has countable conjugacy classes.

Proof. Let $\mathfrak{C}$ be an $\omega$-cover of subgroups of the group $G$ such that each $X \in \mathfrak{C}$ is a totally imprimitive subgroup of $\mathrm{FGL}_{\mathbb{F}_{X}}\left(V_{X}\right)$ for some infinite-dimensional $\mathbb{F}_{X^{-}}$ vector space $V_{X}$. From Lemma 3.2 we may assume that the characteristic $p$ of the fields $\mathbb{F}_{X}$ is independent of $X \in \mathfrak{C}$. Since the unipotent radical of every $X \in \mathfrak{C}$ is trivial, $G$ has no non-trivial normal $p$-subgroup in the case when $p>0$. 
Consider a fixed $X \in \mathfrak{C}$. Let $P_{X}=\{Y \in \mathfrak{C} \mid X \leq Y\}$, and consider some $Y \in P_{X}$. From [1] Proposition 1], the group $X$ cannot be a subdirect product of linear groups. Hence $V_{Y}$ is an irreducible $\mathbb{F}_{Y}\left\langle X^{Y}\right\rangle$-module, and $Y^{\prime} \leq\left\langle X^{Y}\right\rangle$. This holds for every $Y \in P_{X}$, whence $G^{\prime} \leq\left\langle X^{G}\right\rangle$. Let $H=\left\langle X^{G}\right\rangle$. We now show that $H$ is countable.

Let $\left\{X_{n}\right\}_{n \in \omega}$ be an ascending chain of finite subgroups with union $X$. By Lemma 3.2 , we can recursively choose integers $d_{n}(n \in \omega)$ and a descending chain $\left\{\mathfrak{C}_{n}\right\}_{n \in \omega}$ of $\omega$-subcovers of $\mathfrak{C}$ such that

$$
\operatorname{dim}_{\mathbb{F}_{Y}}\left[V_{Y}, X_{n}\right] \leq d_{n} \quad \text { for all } Y \in \mathfrak{C}_{n}, n \in \omega .
$$

Let $\Phi_{n}$ be an ultrafilter on $\mathfrak{C}_{\mathfrak{n}}$ which contains the set $P_{Y} \cap \mathfrak{C}_{n}$ for every $Y \in \mathfrak{C}_{n}$. For each $n \in \omega$, the group $G$ embeds into the ultraproduct $K_{n}=\left(\prod_{Y \in \mathfrak{C}_{n}} Y\right) / \Phi_{n}$ via $g=\left(g_{Y}\right)_{Y \in \mathfrak{C}_{n}}$ for all $g \in G$; here $g_{Y}=g$ if $g \in Y$, and $g_{Y}=1$ otherwise. From Łoś Theorem, $K_{n}$ acts as a transformation group on the vector space $W_{n}=$ $\left(\prod_{Y \in \mathfrak{C}_{n}} V_{Y}\right) / \Phi_{n}$ in such a way, that

$$
\operatorname{dim}\left[W_{n}, X_{m}\right] \leq d_{m} \text { for all } m \leq n .
$$

Let $\Psi$ be an ultrafilter on $\omega$ containing all cofinite subsets of $\omega$. Then $G$ embeds diagonally into the ultraproduct $K=\left(\prod_{n \in \omega} K_{n}\right) / \Psi$, which acts as a transformation group on the vector space $W=\left(\prod_{n \in \omega} W_{n}\right) / \Psi$ in such a way, that

$$
\operatorname{dim}\left[W, X_{n}\right] \leq d_{n} \text { for all } n \in \omega .
$$

In particular, $H \leq \mathrm{FGL}(W)$. Let $\mathfrak{S}$ be an $H$-composition series in $W$. As in the proof of [16, Theorem B(vi)], each of the normal closures $\left\langle X_{n}^{G}\right\rangle=\left\langle X_{n}^{G^{\prime}}\right\rangle=\left\langle X_{n}^{H}\right\rangle$ $(n \in \omega)$ acts non-trivially on just finitely many factors of $\mathfrak{S}$. Therefore $H$ acts nontrivially on just countably many factors of $\mathfrak{S}$. Since the characteristic of the field $\mathbb{F}$ associated with $W$ is still $p$, the unipotent radical of $H$ is trivial. Therefore $H$ is a subdirect product of the countably many groups induced from $H$ on the above factors of $\mathfrak{S}$. Since we want to prove countability of $H$, it suffices to consider the action of $H$ on one of these factors; that is, we may assume without loss that $H$ acts irreducibly on $W$. If $\operatorname{dim}_{\mathbb{F}} W$ is finite, then $H$ is countable from [23, 1.19] and 28], 27. Suppose now that $\operatorname{dim}_{\mathbb{F}} W$ is infinite. Since no $Y \in \mathfrak{C}$ contains an infinite alternating section, the same holds for $H$. Hence $H$ is a totally imprimitive subgroup of $\mathrm{FGL}_{\mathbb{F}}(W)$. In particular, $H$ is countable.

Our next result corresponds to [7, Theorem 4].

Proposition 6.4. Totally imprimitive periodic finitary linear groups without nontrivial abelian normal subgroups are countably recognizable.

Proof. Let the periodic group $G$ have an $\omega$-cover of totally imprimitive finitary linear groups which do not contain a non-trivial abelian normal subgroup. By Proposition 6.3, the derived subgroup of $G$ is countable, and hence an irreducible subgroup of some group in the $\omega$-cover. Thus $G^{\prime}$ is a totally imprimitive subgroup of $\mathrm{FGL}_{\mathbb{F}}(V)$ for some infinite-dimensional $\mathbb{F}$-vector space $V$. From [12] we may choose $\mathbb{F}$ algebraically closed. Consider a non-trivial finite subgroup $F$ of $G^{\prime}$. Choose a proper $G^{\prime}$-block $V_{0}$ in $V$ such that $F$ fixes each $V_{0} g \neq V_{0} \quad\left(g \in G^{\prime}\right)$ elementwise. Then $C_{G^{\prime}}\left(F^{G}\right)$ must leave each $V_{0} g\left(g \in G^{\prime}\right)$ invariant. From [23, 1.17] the normalizer in $G^{\prime}$ of $V_{0}$ contains a finite subgroup $E$ such that $\mathbb{F} E$ induces $\operatorname{End}_{\mathbb{F}}\left(V_{0}\right)$ on $V_{0}$. From Schur's Lemma $([23,1.2])$, the centralizer $C_{G^{\prime}}\left(\langle E, F\rangle^{G}\right)$ acts like a scalar on each $V_{0} g\left(g \in G^{\prime}\right)$. The hypothesis now yields $C_{G^{\prime}}\left(\langle E, F\rangle^{G}\right)=1$, whence $C_{G}\left(\langle E, F\rangle^{G}\right)=1$ too. Since $G$ has countable conjugacy classes (Proposition 6.3), 
the centralizer $C_{G}\left(\langle E, F\rangle^{G}\right)$ has countable index in $G$. And so $G$ must be countable.

Theorem 6.5. Theorem $C$ holds for the class $\mathfrak{X}$ of all irreducible periodic finitary linear groups.

Proof. Let $\mathfrak{C}$ be an $\omega$-cover of the group $G$ such that each $X \in \mathfrak{C}$ is a totally imprimitive subgroup of $\mathrm{FGL}_{\mathbb{F}_{X}}\left(V_{X}\right)$ for some infinite-dimensional $\mathbb{F}_{X}$-vector space $V_{X}$. From Lemma 3.2 we may assume that $\chi=\operatorname{char} \mathbb{F}_{X}$ is independent of $X \in \mathfrak{C}$. And [12] allows us to choose for $\mathbb{F}_{X}$ the algebraic closure $\mathbb{F}$ of the prime field in characteristic $\chi$. By Proposition 6.3, the commutator subgroup $G^{\prime}$ is countable. We may therefore assume that $G^{\prime}=X^{\prime}$ for all $X \in \mathfrak{C}$.

From Proposition 6.4 we may assume that $G$ contains a non-trivial elementaryabelian normal $p$-subgroup $N$ for some prime $p$. Since every $X \in \mathfrak{C}$ has trivial unipotent radical, we see that $p \neq \chi$. Fix $1 \neq z \in N \cap G^{\prime}$. For every $X \in \mathfrak{C}$ there exists an $X$-block $V_{X, 0}$ in $V_{X}$ such that $z$ acts just on $V_{X, 0}$, that is, $z$ centralizes every $V_{X, 0} x \neq V_{X, 0}(x \in X)$. As $N$ is elementary-abelian, $z$ has just finitely many $G$-conjugates which act just on $V_{X, 0}[23,2.2$. Since $z$ has just countably many $G$-conjugates, Lemma 3.2 allows us to assume that $d=\operatorname{dim}_{\mathbb{F}} V_{X, 0}$ and the finite set of those $G$-conjugates of $z$ which act just on $V_{X, 0}$ are independent of $X \in \mathfrak{C}$. Let $F$ be their join, and let $F=F_{0}, F_{1}, \ldots$.. be the $G$-conjugates of $F$. From Schur's Lemma [23, 1.2], the elementary-abelian $p$-group $\left\langle F^{G}\right\rangle$ acts like a scalar on each of its homogeneous components. Therefore the $G$-blocks

$$
V_{X, i}=\left\{v \in V_{X} \mid\left[v, F_{i}\right] \neq 0\right\} \cup\{0\} \quad(i \in \omega)
$$

form a system of imprimitivity in $V_{X}$. Let $V_{0}=\mathbb{F}^{d}$ and $V=V_{0} \otimes \omega$. An identification of $V_{X, i}$ with $V_{0} \otimes i$ leads to an identification of $V_{X}$ with $V$, and to an embedding $\sigma_{X}$ of $X$ into the restricted wreath product $\mathrm{GL}_{\mathbb{F}}\left(V_{0}\right) \operatorname{wr}_{\omega} \mathrm{FSym}(\omega)$, which acts on $V$. For each $g \in X \in \mathfrak{C}$ we have $g \sigma_{X}=f_{X, g} \pi_{g}$ for some $f_{X, g}: \omega \rightarrow$ $\mathrm{GL}_{\mathbb{F}}\left(V_{0}\right)$, where $\pi_{g} \in \mathrm{FSym}(\omega)$ is given via $F_{i \pi_{g}}=F_{i}^{g}$, independently of $X \in \mathfrak{C}$. We let $\pi$ denote the corresponding homomorphism $g \mapsto \pi_{g}$ of $G$ into $\operatorname{FSym}(\omega)$.

Now $\omega$ contains just countably many proper $G \pi$-blocks $\Omega_{n}(n \in \omega)$. The normalizer in $G^{\prime} \sigma_{X}$ of $V_{0} \otimes \Omega_{n}$ acts irreducibly on $V_{0} \otimes \Omega_{n}$, and $\mathbb{F}$ is algebraically closed. Hence [23, 1.17] and Lemma 3.2 yield a sequence $\left\{E_{n}\right\}_{n \in \omega}$ of finite subgroups of $G^{\prime}$, and a descending chain $\left\{\mathfrak{C}_{n}\right\}_{n \in \omega}$ of $\omega$-subcovers of $\mathfrak{C}$ such that $\mathbb{F}\left(E_{n} \sigma_{X}\right)$ induces $\operatorname{End}_{\mathbb{F}}\left(V_{0} \otimes \Omega_{n}\right)$ on $V_{0} \otimes \Omega_{n}$ for all $X \in \mathfrak{C}_{n}, n \in \omega$. Since $V$ is countable, we may even assume that $\left.\sigma_{X}\right|_{E_{n}}=\left.\sigma_{Y}\right|_{E_{n}}$ for all $X, Y \in \mathfrak{C}_{n}, n \in \omega$.

Next, let $\Phi_{n}$ be an ultrafilter on $\mathfrak{C}_{n}$ which contains the set $P_{X} \cap \mathfrak{C}_{n}$ for every $X \in \mathfrak{C}$, where $P_{X}=\{Y \in \mathfrak{C} \mid X \leq Y\}$. From Loś Theorem, the vector space $U_{n}=\left(\prod_{X \in \mathfrak{C}_{n}} V_{0}\right) / \Phi_{n}$ over the algebraically closed field $\mathbb{K}_{n}=\left(\prod_{X \in \mathfrak{C}_{n}} \mathbb{F}\right) / \Phi_{n}$ has dimension $d$. And a group homomorphism $\tau_{n}$ of $G$ into the unrestricted wreath product $W_{n}=\operatorname{GL}_{\mathbb{K}_{n}}\left(U_{n}\right) \operatorname{Wr}_{\omega} \operatorname{FSym}(\omega)$ is given by $g \tau_{n}=f_{g}^{n} \pi_{g}$ for all $g \in G$, where $f_{g}^{n}: \omega \rightarrow \mathrm{GL}_{\mathbb{K}_{n}}\left(U_{n}\right)$ is defined via

$$
(i) f_{g}^{n}=\left((i) f_{X, g}\right)_{X \in \mathfrak{C}_{n}} \text { for all } i \in \omega ;
$$

here $f_{X, g}=1$ whenever $g \notin X \in \mathfrak{C}_{n}$. We shall show next that each $\tau_{n}$ is actually an embedding.

Assume that $\operatorname{ker} \tau_{n} \neq 1$. Clearly $\operatorname{ker} \tau_{n}$ is not a $\chi$-group. Choose $1 \neq g \in$ $G^{\prime} \cap \operatorname{ker} \tau_{n}$ of prime order $q \neq \chi$. Then $\pi_{g}=1$, and $g \sigma_{X}\left(X \in \mathfrak{C}_{n}\right)$ normalizes each block $V_{0} \otimes i(i \in \omega)$. The elementary-abelian $q$-subgroups of $\mathrm{GL}_{\mathbb{F}}\left(V_{0} \otimes i\right)$ 
have ranks bounded by $d([23,2.2])$. Choose positive integers $k<\ell$ such that the holomorph of the elementary-abelian $q$-group of rank $d$ satisfies the identity $[x, \ell y]=[x, \ell-k y]$ (Lemma 6.1). Note that $\ell \geq d$. From [17, Lemma 3.2] there exists $h \in G^{\prime}$ such that the elements $h^{-j} g h^{j}(0 \leq j \leq \ell)$ commute, since they act just on $X$-blocks of $V_{X}$ with pairwise trivial intersections, for some $X \in \mathfrak{C}$. Lemma 6.2 yields $\left[g_{, \ell} h\right] \neq\left[g_{\ell-k} h\right]$. Let $\omega_{0}=\operatorname{supp}_{\omega} \pi_{h}$. For fixed $X \in \mathfrak{C}$ and $i \in \omega-\omega_{0}$, let $\eta_{i}$ denote the projection of $\langle g, h\rangle$ into $\operatorname{GL}_{\mathbb{F}}\left(V_{X, i}\right)$. Since $\left(h^{-k} g h^{k}\right) \eta_{i} \in A_{i}=$ $\left\langle h^{-j} g h^{j} \mid 0 \leq j<k\right\rangle \eta_{i}$ for some $k \leq d$, the elementary-abelian $q$-group $A_{i}$ is $h \eta_{i^{-}}$ invariant. By choice of $k$ and $\ell$ we have $\left([g, \ell h] \cdot\left[g_{, \ell-k} h\right]^{-1}\right) \eta_{i}=1$. It follows that $\left([g, \ell h] \cdot\left[g_{, \ell-k} h\right]^{-1}\right) \sigma_{X}$ acts just on $V_{0} \otimes \omega_{0}$, for all $X \in \mathfrak{C}_{n}$. Hence, for every $X \in \mathfrak{C}$ there is some $i \in \omega_{0}$ such that $(i) f_{X, g} \neq 1$. By [5, Lemma B.1] there exists $i \in \omega_{0}$ such that $\left\{X \in \mathfrak{X} \mid(i) f_{X, g} \neq 1\right\} \in \Phi$. But then $(i) f_{g}^{n} \neq 1$, and hence $g \tau_{n} \neq 1$, a contradiction. Thus, $\tau_{n}$ is an embedding of $G$ into $W_{n}$.

Let $\Psi$ be an ultrafilter on $\omega$ containing all cofinite subsets of $\omega$. Again, $U=$ $\left(\prod_{n \in \omega} U_{n}\right) / \Psi$ is a $d$-dimensional vector space over the algebraically closed field $\mathbb{K}=\left(\prod_{n \in \omega} \mathbb{K}_{n}\right) / \Psi$, and an embedding $\tau: G \longrightarrow W=\operatorname{GL}_{\mathbb{K}}(U) \operatorname{Wr}_{\omega} \operatorname{FSym}(\omega)$ is given by $g \tau=f_{g} \pi_{g}$ for all $g \in G$, where

$$
(i) f_{g}=\left((i) f_{g}^{n}\right)_{n \in \omega} \text { for all } i \in \omega .
$$

Since $G$ does not contain an infinite alternating section, the image $G \pi$ of the projection of $G$ into the top group of $W$ is a totally imprimitive subgroup of $\mathrm{FSym}(\omega)$. It remains to show that $G \tau$ acts irreducibly on $U \otimes \omega$, and that the base group functions $f_{g}(g \in G)$ act like scalars on $U \otimes \Delta$ for almost all $G \pi$-translates $\Delta$ of every $\Omega_{n}(n \in \omega)$.

First of all we identify $\mathbb{F}$ diagonally with a subfield of each $\mathbb{K}_{n}(n \in \omega)$ and of $\mathbb{K}$. Similarly we can identify $\mathbb{K}_{n} \otimes V_{0}$, resp. $\mathbb{K} \otimes V_{0}$, with a $\mathbb{K}_{n^{-}}$resp. $\mathbb{K}$-subspace of $U_{n}$, resp. $U$. In this way all the embeddings $\sigma_{X}(X \in \mathfrak{C})$ map into each $W_{n}$ $(n \in \omega)$ and into $W$. From our choice of $E_{n}$ we see that $\left.\tau_{n}\right|_{E_{n}}=\left.\sigma_{X}\right|_{E_{n}}$ for all $n$ and all $X \in \mathfrak{C}_{n}$, whence $\left.\tau\right|_{E_{n}}=\left.\sigma_{X}\right|_{E_{n}}$. Since $\left.\operatorname{dim}_{\mathbb{K}} \mathbb{K}\left(E_{n} \tau\right)\right|_{U \otimes \Omega_{n}}=\left(d \cdot\left|\Omega_{n}\right|\right)^{2}$, we conclude that $\mathbb{K}\left(E_{n} \tau\right)$ induces $\operatorname{End}_{\mathbb{K}}\left(U \otimes \Omega_{n}\right)$ on $U \otimes \Omega_{n}$. In particular, $G \tau$ is an irreducible subgroup of $W$.

Now consider fixed $g \in G, n \in \omega$. Choose $X \in \mathfrak{C}_{n}$ containing $g$. Then $\Omega_{n}$ is contained in a proper $G \pi$-block $\bar{\Omega}_{n}$ of $\omega$ such that $\left\langle E_{n}, g\right\rangle \sigma_{X}$ acts trivially on $U \otimes\left(\omega-\bar{\Omega}_{n}\right)$. Note that $\bar{\Omega}_{n}$ contains just finitely many $G \pi$-translates of $\Omega_{n}$. Consider a $G \pi$-translate $\Delta$ of $\Omega_{n}$ which lies in $\omega-\bar{\Omega}_{n}$. Then there exists $h \in G^{\prime}$ such that $E_{n}^{h} \sigma_{X}$ and $E_{n}^{h} \tau$ act trivially on $U \otimes \bar{\Omega}_{n}$, and such that $\mathbb{K}\left(E_{n}^{h} \sigma_{X}\right)$ and $\mathbb{K}\left(E_{n}^{h} \tau\right)$ induce $\operatorname{End}_{\mathbb{K}}(U \otimes \Delta)$ on $U \otimes \Delta$. Since $g \sigma_{X}$ commutes with $E_{n}^{h} \sigma_{X}$, also $g \tau$ commutes with $E_{n}^{h} \tau$. But $\operatorname{supp}_{\omega} \pi_{g} \subseteq \bar{\Omega}_{n}$. Therefore $\left.f_{g}\right|_{U \otimes \Delta} \in \mathrm{GL}_{\mathbb{K}}(U \otimes \Delta)$ commutes with $\left.\mathbb{K}\left(E_{n}^{h} \tau\right)\right|_{U \otimes \Delta}=\operatorname{End}_{\mathbb{K}}(U \otimes \Delta)$. We thus obtain that $f_{g}$ acts like a scalar on $U \otimes \Delta$.

The permutation group version of Theorem $\mathrm{C}$ will be proved similarly to the transformation group version. For the convenience of the reader we first give a proof of the crucial [7, Lemma 1].

Proposition 6.6 ([7 Lemma 1]). Let the group $G$ have an $\omega$-cover of totally imprimitive finitary permutation groups. Then the commutator subgroup $G^{\prime}$ of $G$ is countable. 
Proof. Let $\mathfrak{C}$ be an $\omega$-cover of subgroups of the group $G$ such that each $X \in \mathfrak{C}$ is a totally imprimitive subgroup of $\operatorname{FSym}\left(\Omega_{X}\right)$ for some infinite set $\Omega_{X}$. For fixed $X \in \mathfrak{C}$, let $P_{X}=\{Y \in \mathfrak{C} \mid X \leq Y\}$, and consider some $Y \in P_{X}$. From [25. Theorem 1], the group $X$ cannot be a subdirect product of finite groups. Hence $\left\langle X^{Y}\right\rangle$ acts transitively on $\Omega_{X}$, and $Y^{\prime} \leq\left\langle X^{Y}\right\rangle$. This holds for every $Y \in P_{X}$, whence $G^{\prime} \leq\left\langle X^{G}\right\rangle$. Therefore it suffices to show that $G$ has countable conjugacy classes.

Consider elements $g, h \in G$. If $g, h \in X \in \mathfrak{C}$, then $\operatorname{supp}_{\Omega_{X}}\langle g, h\rangle$ is contained in a finite $X$-block in $\Omega_{X}$, whence $h$ centralizes all but finitely many $X$-conjugates of $g$. An application of Lemma 3.2 shows that $h$ centralizes all but finitely many $G$-conjugates of $g$. In particular, $G$ acts finitarily on the conjugacy class $g^{G}$. Since no $X \in \mathfrak{C}$ has an infinite alternating section, the same holds for $G$. But then $G$ must act as a totally imprimitive finitary permutation group on $g^{G}$, whence $g^{G}$ is countable.

Theorem 6.7. Theorem $C$ holds for the class $\mathfrak{X}$ of all transitive finitary permutation groups.

Proof. Let $\mathfrak{C}$ be an $\omega$-cover of the group $G$ such that each $X \in \mathfrak{C}$ is a totally imprimitive subgroup of $\operatorname{FSym}\left(\Omega_{X}\right)$ for some infinite set $\Omega_{X}$. By Proposition 6.6, the commutator subgroup $G^{\prime}$ is countable. We may therefore assume that $G^{\prime}=X^{\prime}$ for all $X \in \mathfrak{C}$.

Fix $1 \neq z \in G^{\prime}$. For every $X \in \mathfrak{C}$ there exists an $X$-block $\Omega_{X, 0}$ in $\Omega_{X}$ such that $z$ fixes $\Omega_{X}-\Omega_{X, 0}$ pointwise. Since $G^{\prime}$ is countable, Lemma 3.2 allows us to assume that $d=\left|\Omega_{X, 0}\right|$ and the finite set of those $G$-conjugates of $z$ that fix $\Omega_{X}-\Omega_{X, 0}$ pointwise are independent of $X \in \mathfrak{C}$. Let $F$ be their join, and let $F=F_{0}, F_{1}, \ldots$. be the $G$-conjugates of $F$. For every $G$-translate $\Delta$ of $\Omega_{X, 0}$ there exists a unique $i$ such that $\operatorname{supp}_{\Omega_{X}} F_{i} \subseteq \Delta$; we denote $\Delta$ by $\Omega_{X, i}$. Let $\Gamma=\{1, \ldots, d\}$ and $\Omega=\Gamma \times \omega$. An identification of $\Omega_{X, i}$ with $\Gamma \times i$ leads to an identification of $\Omega_{X}$ with $\Omega$, and to an embedding $\sigma_{X}: X \longrightarrow \operatorname{Sym}(\Gamma){ }_{w_{\omega}} \operatorname{FSym}(\omega)$, where the wreath product acts on $\Omega$. For each $g \in X \in \mathfrak{C}$ we have $g \sigma_{X}=f_{X, g} \pi_{g}$ for some $f_{X, g}: \omega \rightarrow \operatorname{Sym}(\Gamma)$, where $\pi_{g} \in \operatorname{FSym}(\omega)$ is given via $F_{i \pi_{g}}=F_{i}^{g}$, independently of $X \in \mathfrak{C}$. We let $\pi$ denote the corresponding homomorphism $g \mapsto \pi_{g}$ of $G$ into $\operatorname{FSym}(\omega)$.

Now $\omega$ contains just countably many proper $G \pi$-blocks $\Omega_{n}(n \in \omega)$. The setwise stabilizer $S_{X, n} \sigma_{X}$ in $G^{\prime} \sigma_{X}$ of $\Gamma \times \Omega_{n}$ acts transitively on $\Gamma \times \Omega_{n}$. Hence Lemma 3.2 yields a sequence $\left\{E_{n}\right\}_{n \in \omega}$ of finite subgroups of $G^{\prime}$, and a descending chain $\left\{\mathfrak{C}_{n}\right\}_{n \in \omega}$ of $\omega$-subcovers of $\mathfrak{C}$ such that $E_{n} \sigma_{X}$ induces the action of $S_{X, n} \sigma_{X}$ on $\Gamma \times \Omega_{n}$, for all $X \in \mathfrak{C}_{n}, n \in \omega$. Since $\Omega$ is countable, we may even assume that $\left.\sigma_{X}\right|_{E_{n}}=\left.\sigma_{Y}\right|_{E_{n}}$ for all $X, Y \in \mathfrak{C}_{n}, n \in \omega$.

In exactly the same way as in the proof of Theorem 6.5 we can now invoke ultraproduct constructions to obtain an embedding $\tau: G \longrightarrow S \operatorname{Wr}_{\omega} \operatorname{FSym}(\Omega)$ with the desired properties; here $S$ is the subgroup of $\operatorname{Sym}\left(\Gamma \times \Omega_{0}\right)$ induced from $E_{0} \sigma_{X}$.

\section{Some Sufficient Conditions}

This section consists of a brief discussion of sufficient conditions for countable recognizability of totally imprimitive $\mathfrak{X}$-groups. It shows how Theorem $\mathrm{C}$ can be applied in particular instances. The following observation is straightforward from Theorem $\mathrm{C}$ and generalizes conditions given in [7] for the permutation group case. 
Proposition 7.1. The class of all totally imprimitive $\mathfrak{X}$-groups $G$ that satisfy $\left|N G^{\prime} / G^{\prime}\right|<\infty$ for every elementary-abelian normal subgroup $N$ is countably recognizable.

Proof. Assume that there exists an uncountable group $G$ with an $\omega$-cover $\mathfrak{C}$ of totally imprimitive $\mathfrak{X}$-subgroups satisfying the property in the statement of the proposition. Then $G$ has an uncountable abelian normal subgroup $N$ by Theorem C. We can replace $G$ by $G^{\prime} N$ and choose $N$ elementary-abelian. Lemma 3.2 allows us to assume that the finite value $s=\left|(N \cap X) G^{\prime} / G^{\prime}\right|$ is independent of $X \in \mathfrak{C}$. But then $\left|N G^{\prime} / G^{\prime}\right|=s$ too, in contradiction to the uncountability of $N$.

Remark 7.2. Among the totally imprimitive $\mathfrak{X}$-groups $G$ satisfying $\left|N G^{\prime} / G^{\prime}\right|<$ $\infty$ for every elementary-abelian normal subgroup $N$ are not only those without abelian normal subgroups, but also full wreath products $L$ wr $F_{1}$ wr $\cdots$ wr $F_{n}$ wr $\cdots$, where the $F_{n}$ are finite groups, and where $L$ is a finite resp. irreducible linear group. In fact, for every elementary-abelian normal subgroup $N$ of such a full wreath product $G$ there exists $n \in \omega$ such that $N$ is contained in $\left\langle A^{G}\right\rangle$, where $A$ is an elementary-abelian normal subgroup of the unipotent-free linear group $L_{n}=L \mathrm{wr} F_{1} \mathrm{wr} \cdots \mathrm{wr} F_{n}$; and it is well-known that $\left\langle A^{G}\right\rangle G^{\prime} / G^{\prime} \cong A /\left[A, L_{n}\right]$ is finite.

Our most spectacular result, however, concerns $p$-groups.

Proof of Theorem D. Assume that there exists an uncountable $p$-group $G$ with an $\omega$-cover of totally imprimitive $\mathfrak{X}$-subgroups. Then $G$ has an uncountable abelian normal subgroup $N$ by Theorem C. We can replace $G$ by $G^{\prime} N$ and choose $N$ elementary-abelian. Now we refer to the notation introduced in the proofs of Theorems 6.5 and 6.7 , and we choose $\Omega_{0}=\{0\}$. In addition, Lemma 3.2 allows us to assume that $\left.\sigma_{X}\right|_{F}=\left.\sigma_{Y}\right|_{F}$ for all $X, Y \in \mathfrak{C}$.

We consider the transformation group case first. Since $F \sigma_{X}$ acts just on $V_{0} \otimes 0$, it is normalized by $E_{0} \sigma_{X}\left(X \in \mathfrak{C}_{0}\right)$. Because $G$ is a $p$-group, the centralizer $C_{F}\left(E_{0}\right)$ must be non-trivial. Let $T$ be a right transversal in $G^{\prime}$ of the setwise stabilizer in $G^{\prime}$ of $V_{0} \otimes 0$. For all $t \in T, X \in \mathfrak{C}_{0}$, the group algebra $\mathbb{F}\left(E_{0}^{t} \sigma_{X}\right)$ induces $\operatorname{End}_{\mathbb{F}}\left(V_{0} \otimes 0 \pi_{t}\right)$ on $V_{0} \otimes 0 \pi_{t}$. Therefore, $\left(C_{N \cap X}\left(E_{0}^{t}\right)\right) \sigma_{X}$ acts like $\left(C_{F}\left(E_{0}\right)^{t}\right) \sigma_{X}$ on $V_{0} \otimes 0 \pi_{t}$. It follows that

$$
C_{N \cap X}\left(E_{0}^{G}\right) \leq \operatorname{Dr}_{t \in T}\left(C_{F}\left(E_{0}\right)\right)^{t} \leq C_{F}\left(E_{0}\right) \cdot\left[F, G^{\prime}\right] \leq F \cdot G^{\prime}
$$

for all $X \in \mathfrak{C}_{0}$. And so $C_{N}\left(E_{0}^{G}\right)$ becomes countable. On the other hand, the conjugacy classes in $G$ are countable, so $C_{N}\left(E_{0}^{G}\right)$ has countable index in $N$. This contradicts the uncountability of $N$.

We now consider the permutation group case. From Lemma 3.2 we may assume that the subgroup of $\operatorname{Sym}(\Gamma \times 0)$ induced from $E_{0} \sigma_{X}$ is independent of $X \in \mathfrak{C}_{0}$, so that it can be identified with $S$. In this way the embeddings $\sigma_{X}$ $\left(X \in \mathfrak{C}_{0}\right)$ and $\tau_{0}$ map into the wreath product $W=S \operatorname{Wr}_{\omega} \operatorname{FSym}(\omega)$. The augmentation ideal of the group algebra $\mathbb{C} S$ is a direct sum of non-trivial irreducible $\mathbb{C} S$-submodules $M_{1}, \ldots, M_{r}$, and there is a faithful action of $W$ on the $\mathbb{C}$-vector space $\bigoplus_{i=1}^{r}\left(M_{i} \otimes \omega\right)$. In particular, $W$ is a subdirect product of the wreath products $Z_{i}=\operatorname{GL}_{\mathbb{C}}\left(M_{i}\right) \operatorname{Wr}_{\omega} \operatorname{FSym}(\omega)(1 \leq i \leq r)$, and the projection $\rho_{j}: W \rightarrow Z_{j}$ must map $N \tau_{0}$ onto an uncountable subgroup of $Z_{j}$ for some fixed $j$. Let $\eta=\tau_{0} \rho_{j}$. Since the conjugacy classes in $G \eta$ are countable too, it again suffices to show that $C_{N \eta}\left(\left(E_{0}^{G}\right) \eta\right)$ is countable. To this end we can copy the argument applied in the 
transformation group case: We may assume that $F \eta$ is non-trivial. Then $F \eta$ acts just on $M_{j} \otimes 0$ and is therefore normalized by $E_{0} \eta$. Thus $C_{F \eta}\left(E_{0} \eta\right)$ is non-trivial. And since $E_{0} \tau_{0}$ induces the action of $S$ on $S \times 0$, the group $E_{0} \eta$ acts irreducibly on $M_{j} \otimes 0$, whence $C_{(N \cap X) \eta}\left(\left(E_{0}^{t}\right) \eta\right)$ acts like $C_{F \eta}\left(E_{0} \eta\right)^{t \eta}$ on $M_{j} \otimes 0 \pi_{t}$, for all $X \in \mathfrak{C}_{0}, t \in G^{\prime}$.

Another observation deals with the coprime situation.

Proposition 7.3. Suppose that in Theorem $C$ the orders of the elements in $C \cap$ $G$ are coprime to the orders of the elements in $G / C \cap G$. Then $G$ is a totally imprimitive $\mathfrak{X}$-group.

Proof. Assume that there exists an uncountable group $G$ as in Theorem $\mathrm{C}$ such that the elements in $N=C \cap G$ have orders coprime to those of the elements in $G / N$. Without loss we may assume that $G=G^{\prime} N$, and that $N$ is an elementary-abelian $p$-group for some prime $p$. Since $N$ is uncountable, we can find $z \in N-[N, G]$. In order to copy the argument in the proof of Theorem D, we just need to show that $C_{F}\left(E_{0}\right) \neq 1$. But because of the coprime situation we have $F=\left[F, E_{0}\right] \times C_{F}\left(E_{0}\right)$; and since $z \in F-\left[F, E_{0}\right]$, we see that $C_{F}\left(E_{0}\right) \neq 1$.

We shall conclude with a quite interesting condition on stabilizers of blocks.

Proposition 7.4. Consider the situation in Theorem $C$, and let $H$ denote the setwise stabilizer in $G^{\prime}$ of $S \times 0$ resp. $U \otimes 0$. If $\operatorname{Hom}_{\mathbb{Z}}\left(H, \zeta_{1}(S)\right)$ is countable, then $G$ is a totally imprimitive $\mathfrak{X}$-group.

Proof. We refer to the notation introduced in Theorem $\mathrm{C}$ and in the proofs of Theorems 6.5/6.7, and we choose $\Omega_{0}=\{0\}$. As in the proof of Theorem $D$, the embeddings $\sigma_{X}\left(X \in \mathfrak{C}_{0}\right)$ map into $W=S \operatorname{Wr}_{\omega} \operatorname{FSym}(\omega)$. Fix some $X \in \mathfrak{C}_{0}$. Let $K$ denote the subgroup of $W$ which consists of all base group functions $\omega \rightarrow \zeta_{1}(S)$. For every $Y \in \mathfrak{C}_{0}$, the difference map $\delta_{Y, X}$ of $G^{\prime}$ into the base group of $W$ is defined via

$$
g \delta_{Y, X}=\left(g \sigma_{Y}\right)^{-1} \cdot g \sigma_{X} \quad \text { for all } g \in G^{\prime} .
$$

Consider $h \in E_{0}$ and $t \in G^{\prime}$. Because $\left.\sigma_{X}\right|_{E_{0}}=\left.\sigma_{Y}\right|_{E_{0}}$, the element $t \delta_{Y, X}$ centralizes $E_{0} \sigma_{X}$. Hence

$$
\left(h^{t^{-1}}\right) \sigma_{X}=\left(h \sigma_{X}\right)^{\left(t \sigma_{X}\right)^{-1}}=\left(h \sigma_{X}\right)^{t \delta_{Y, X} \cdot\left(t \sigma_{X}\right)^{-1}}=\left(h \sigma_{Y}\right)^{\left(t \sigma_{Y}\right)^{-1}}=\left(h^{t^{-1}}\right) \sigma_{Y},
$$

that is, $\left.\sigma_{X}\right|_{\left\langle E_{0}^{G^{\prime}}\right\rangle}=\left.\sigma_{Y}\right|_{\left\langle E_{0}^{G^{\prime}}\right\rangle}$. Since $E_{0}^{t} \sigma_{X}$ induces the action of $S$ on $S \times 0 \pi_{t}$, resp. since $\mathbb{K}\left(E_{0}^{t} \sigma_{X}\right)$ induces the action of $\operatorname{End}_{\mathbb{K}}(U)$ on $U \otimes 0 \pi_{t}$, we obtain $G^{\prime} \delta_{Y, X} \leq K$. Thus $\delta_{Y, X}$ is a derivation of $G^{\prime}$ into the $G \pi$-module $K$, that is,

$$
(g h) \delta_{Y, X}=\left(g \delta_{Y, X}\right)^{h \pi} \cdot h \delta_{Y, X} \quad \text { for all } g, h \in G^{\prime} .
$$

Consider $\zeta_{1}(S)$ as a trivial $\mathbb{Z} H$-module. Now $H \pi$ is the stabilizer of the point 0 in the transitive subgroup $G^{\prime} \pi$ of $\operatorname{FSym}(\omega)$, and so an isomorphism

$$
\varphi: K \longrightarrow \operatorname{Hom}_{\mathbb{Z} H}\left(\mathbb{Z} G^{\prime}, \zeta_{1}(S)\right)
$$

is given via

$$
(g)(f \varphi)=\left(0 \pi_{g}\right) f \quad \text { for all } f \in K, g \in G^{\prime} .
$$

Therefore Shapiro's Lemma ([9, Section 6.3]) yields $\mathrm{H}^{1}\left(G^{\prime}, K\right) \cong \mathrm{H}^{1}\left(H, \zeta_{1}(S)\right)$. Recall that $\mathrm{H}^{1}\left(G^{\prime}, K\right)$ is isomorphic to the group of outer derivations $G^{\prime} \rightarrow K$ $([22,11.4 .6])$. And every derivation $H \rightarrow \zeta_{1}(S)$ is a homomorphism. By hypothesis 
there are just countably many such homomorphisms. Therefore Lemma 3.2 allows us to assume that there exists a fixed derivation $\delta: G^{\prime} \rightarrow K$ such that $\delta_{Y, X}-\delta$ is an inner derivation for all $Y \in \mathfrak{C}_{0}-\{X\}$. Fix $Y \in \mathfrak{C}_{0}-\{X\}$. Then $\delta_{Z, Y}=\delta_{Z, X}-\delta_{Y, X}$ is an inner derivation for all $Z \in \mathfrak{C}_{0}-\{X, Y\}$, that is, there exists $c_{Z} \in K$ such that

$$
\left(g \sigma_{Z}\right)^{-1} \cdot g \sigma_{Y}=\left[g \pi, c_{Z}\right] \quad \text { for all } g \in G^{\prime} .
$$

Define $\sigma: G \longrightarrow W_{0}=S \operatorname{wr}_{\omega} \operatorname{FSym}(\omega)$ via $g \sigma=g \sigma_{Z} \cdot\left[g \pi, c_{Z}\right]$ whenever $g \in Z \in \mathfrak{C}_{0}-\{X, Y\}$. Since $G^{\prime} \sigma=G^{\prime} \sigma_{Y}$ is an irreducible subgroup of $W_{0}$, it remains to show that $\sigma$ is an embedding.

Suppose that $Z_{1}, Z_{2} \in \mathfrak{C}_{0}-\{X, Y\}$ satisfy $Z_{1} \leq Z_{2}$. Define $\sigma_{i}: Z_{i} \rightarrow W_{0}$ via $g \sigma_{i}=g \sigma_{Z_{i}} \cdot\left[g \pi, c_{Z_{i}}\right]$ for all $g \in Z_{i}(i=1,2)$. Then $\left.\sigma_{1}\right|_{G^{\prime}}=\left.\sigma_{Y}\right|_{G^{\prime}}=\left.\sigma_{2}\right|_{G^{\prime}}$. But $G^{\prime} \sigma_{Y}$ has trivial centralizer in $W_{0}$, whence $\sigma_{1}=\left.\sigma_{2}\right|_{Z_{1}}$. This shows that $\sigma$ is a welldefined homomorphism. $\sigma$ is also injective, since $G^{\prime} \cap \operatorname{ker} \sigma=G^{\prime} \cap \operatorname{ker} \sigma_{Y}=1$.

In the permutation group case, countability of $\operatorname{Hom}_{\mathbb{Z}}\left(H, \zeta_{1}(S)\right)$ in Proposition 7.4 is equivalent to finiteness of $H / H^{p} H^{\prime}$ for every prime $p$ dividing the order of the finite group $\zeta_{1}(S)$.

Corollary 7.5. The class of all totally imprimitive finitary permutation groups $G$ satisfying the following condition is countably recognizable: Whenever $S$ is the normalizer in $G$ of a proper block, and whenever $H$ is the setwise stabilizer in $G$ of the same block, then $H / H^{p} H^{\prime}$ is finite for every prime $p$ dividing the order of some element in $\zeta_{1}(S)$.

Proof. Let $G$ be a group with an $\omega$-cover of subgroups satisfying the hypothesis. Again we refer to the notation introduced in Theorem 6.7. Let $H_{X}(X \in \mathfrak{C})$ denote the setwise stabilizer in $X^{\prime}$ of $S \times 0$. Lemma 3.2 allows us to assume that $\left|H_{X} / H_{X}^{p} H_{X}^{\prime}\right|$ is independent of $X$ for every prime dividing the order of $\zeta_{1}(S)$. The assertion now follows from Theorem $\mathrm{C}$ and Proposition 7.4.

\section{REFERENCES}

[1] B. Bruno and R.E. Phillips, Residual properties of finitary linear groups, J. Algebra 166 (1994), 379-392. MR 95f:20048

[2] J.L. Bell and A. B.Slomson, Models and ultraproducts, North-Holland, Amsterdam, 1969. MR 42:4381

[3] V.V.Belyaev, Finitary linear representations of infinite symmetric and alternating groups, Algebra and Logic 32 (1993), 319-327. MR 95k:20060

[4] J. I. Hall, Finitary linear transformation groups and elements of finite local degree, Arch. Math. 50 (1988), 315-318. MR 89b:20067

[5] J. I. Hall, Locally finite simple groups of finitary linear transformations, Finite and locally finite groups (B. Hartley, G. M. Seitz, A. V. Borovik and R. M. Bryant, eds.), NATO ASI Series C 471, Kluwer Academic Publishers, Dordrecht - Boston - London, 1995, pp. 147-188. MR 96j:20042

[6] J. I. Hall, Periodic simple groups of finitary linear transformations, in preparation.

[7] K. K. Hickin and R. E. Phillips, Some countably stunted finitary permutation groups, preprint.

[8] B. Huppert and N. Blackburn, Finite groups II, Springer Verlag, Berlin - Heidelberg - New York, 1982. MR 84i:20002a

[9] K. W. Gruenberg, Cohomological topics in group theory, Lecture Notes in Math., vol. 143, Springer Verlag, Berlin - Heidelberg - New York, 1970. MR 43:4923

[10] O. H. Kegel and B. A.F. Wehrfritz, Locally finite groups, North-Holland, Amsterdam - London - New York, 1973. MR 57:9848

[11] F. Leinen, Hypercentral unipotent finitary skew linear groups, Comm. Algebra 22 (1994), 929-949. MR 95d:20087 
[12] F. Leinen, Absolute irreducibility for finitary linear groups, Rend. Sem. Mat. Univ. Padova 92 (1994), 59-61. CMP 95:09

[13] F. Leinen and O. Puglisi, Countable recognizability of primitive periodic finitary linear groups, Math. Proc. Cambridge Phil. Soc. 121 (1997), 425-435. MR 98b:20079

[14] A. I. Mal'cev, On faithful representations of infinite groups of matrices, Mat. Sb. 8 (1940), 405-422 (in Russian); English transl., Amer. Math. Soc. Translations (2) 45 (1965), 1-18. MR 2:216d

[15] U. Meierfrankenfeld, Ascending subgroups of irreducible finitary linear groups, J. London Math. Soc. (2) 51 (1995), 75-92. MR 96c:20092

[16] U. Meierfrankenfeld, R. E. Phillips and O. Puglisi, Locally solvable finitary linear groups, J. London Math. Soc. (2) 47 (1993), 31-40. MR 94c:20064

[17] P. M. Neumann, The lawlessness of groups of finitary permutations, Arch. Math. 26 (1975), 561-566. MR 54:406

[18] P. M. Neumann, The structure of finitary permutation groups, Arch. Math. 27 (1976), 3-17. MR 53:5754

[19] R. E. Phillips, The structure of groups of finitary transformations, J. Algebra 119 (1988), 400-448. MR 90a:20080

[20] R. E. Phillips, Finitary linear groups: a survey, Finite and locally finite groups (B. Hartley, G. M. Seitz, A. V. Borovik and R. M. Bryant, eds.), NATO ASI Series C 471, Kluwer Academic Publishers, Dordrecht - Boston - London, 1995, pp. 111-146. MR 96m:20080

[21] D. J.S. Robinson, Finiteness conditions and generalized soluble groups, Vols. 1, 2, Springer Verlag, Berlin - Heidelberg - New York, 1972. [MR 48:11314] MR 48:11315

[22] D. J.S. Robinson, A course in the theory of groups, Springer Verlag, Berlin - Heidelberg New York, 1982. MR 84k:20001

[23] B. A. F. Wehrfritz, Infinite linear groups, Springer Verlag, Berlin - Heidelberg - New York, 1973. MR 49:436

[24] B. A. F. Wehrfritz, On the countable recognition of finitary groups, J. London Math. Soc. (2) 55 (1997), 297-308. MR 98g:20066

[25] J. Wiegold, Groups of finitary permutations, Arch. Math. 25 (1974), 466-469. MR 50:7356

[26] H. Wielandt, Unendliche Permutationsgruppen, Vorlesungen an der Universität Tübingen, $1959 / 60$.

[27] D. J. Winter, Representations of locally finite groups, Bull. Amer. Math. Soc. 74 (1968), 145148. MR 38:3363

[28] A. E. Zalesskii, Maximal periodic subgroups of the full linear group over a field with positive characteristic, Izvestija Akad. Nauk. BSSR, Ser. Fiz.-Mat. Nauk 1966 (1966), 121-123 (in Russian); ZB 252\#20040; MR 33:2732

Fachbereich 17 - Mathematik, Johannes Gutenberg-Universität Mainz, D-55099 Mainz, Germany

Current address: Department of Mathematics, University of Newcastle, Newcastle upon Tyne, NE1 7RU, England

E-mail address: f.a.leinen@ncl.ac.uk

Dipartimento di Matematica, Università degli Studi di Trento, I-38050 Povo (Trento), Italy

E-mail address: puglisi@alpha.science.unitn.it 